\title{
Maternal diesel particle exposure promotes offspring asthma through NK cell-derived granzyme B
}

\author{
Qian Qian, ${ }^{1}$ Bidisha Paul Chowdhury, ${ }^{2}$ Zehua Sun, ${ }^{1}$ Jerica Lenberg, ${ }^{1}$ Rafeul Alam, ${ }^{1,2}$ Eric Vivier, ${ }^{3,4,5}$ and Magdalena M. Gorska ${ }^{1,2}$ \\ 'Division of Allergy and Clinical Immunology, Department of Medicine, National Jewish Health (NJH), Denver, Colorado, USA. 'Division of Allergy and Clinical Immunology, Department of Medicine, \\ University of Colorado Anschutz Medical Campus, Aurora, Colorado, USA. ${ }^{3}$ Innate Pharma Research Labs, Innate Pharma, Marseille, France. ${ }^{4}$ Centre d'Immunologie de Marseille-Luminy, CNRS, INSERM, \\ Aix Marseille University, Marseille, France. ${ }^{5}$ Service d'Immunologie, Marseille Immunopole, Hôpital de la Timone, Assistance Publique des Hôpitaux de Marseille, Marseille, France.
}

\begin{abstract}
Mothers living near high-traffic roads before or during pregnancy are more likely to have children with asthma. Mechanisms are unknown. Using a mouse model, here we showed that maternal exposure to diesel exhaust particles (DEP) predisposed offspring to allergic airway disease ( $A A D$, murine counterpart of human asthma) through programming of their NK cells; predisposition to AAD did not develop in DEP pups that lacked NK cells and was induced in normal pups receiving NK cells from WT DEP pups. DEP NK cells expressed CATA3 and cosecreted IL-13 and the killer protease granzyme $B$ in response to allergen challenge. Extracellular granzyme B did not kill, but instead stimulated protease-activated receptor 2 (PAR2) to cooperate with IL-13 in the induction of IL-25 in airway epithelial cells. Through loss-of-function and reconstitution experiments in pups, we showed that NK cells and granzyme B were required for IL-25 induction and activation of the type 2 immune response and that IL-25 mediated NK cell effects on type 2 response and AAD. Finally, experiments using human cord blood and airway epithelial cells suggested that DEP might induce an identical pathway in humans. Collectively, we describe an NK cell-dependent endotype of AAD that emerged in early life as a result of maternal exposure to DEP.
\end{abstract}

\section{Introduction}

A proportion of patients with asthma develop symptoms of their disease in the first years of life (1). An early inception of a disease is usually linked to aberrant genes. However, genetics is unlikely to be the sole driver of asthma. Gene variants identified by GWAS are considered to account for only a small proportion of asthma prevalence (2). Studies on rare variants are predicted to offer only marginal improvement, leaving the majority of asthma unexplained $(2,3)$. Therefore, it is now acknowledged that development of childhood asthma might be driven in substantial part by environmental exposures that children encounter either in utero or during their first years of life. Environmental impact on childhood asthma was underscored by seminal epidemiological studies that uncovered large differences in prevalence of childhood asthma between countries/regions with more versus less Westernized lifestyles (4, 5). Building on this, other studies showed that childhood asthma positively correlated with exposure to urban air pollution and cigarette smoke and negatively with exposure to the farming environment (6-9). Urban air pollution includes particulate matter (PM) generated by road traffic sources. The major constituents of traffic-related PM (up to 90\%) are diesel exhaust particles (DEP) (6). There is considerable evidence that inhaled DEP directly harm children's lungs, activate their immune systems, and facilitate allergic sensitization and asthma inception $(6,8-10)$. More recent

Conflict of interest: EV is a cofounder and employee of Innate Pharma. Copyright: () 2020, American Society for Clinical Investigation.

Submitted: May 16, 2019; Accepted: April 29, 2020; Published: June 29, 2020

Reference information: J Clin Invest. 2020;130(8):4133-4151.

https://doi.org/10.1172/JCl130324. data emerging from pregnancy cohort studies suggest that DEP may also act prenatally or even preconceptionally and have transgenerational effects $(11,12)$. The risk of childhood asthma increases when mothers live close to major roads and/or in areas with high levels of $\mathrm{PM}_{2.5}(\mathrm{PM} \leq 2.5 \mu \mathrm{m})$ before and/or during pregnancy or when DEP-linked biomarkers are found in the cord blood. This problem is an important one; maternal exposures affect embryos and therefore may induce irreversible developmental damage and/or permanent long-lasting memory in offspring cells and tissues, potentially harming more than postnatal exposures. Prompted by human association studies, we used mouse models to show a causative relationship between maternal exposure to DEP and offspring predisposition to allergic airway disease (AAD, murine counterpart of human asthma) $(13,14)$. Then, using these models, we began to study mechanisms. In this manuscript, we show that susceptibility to AAD in offspring of DEP-exposed mothers is due to emergence of a unique and unexpected pathway linking NK cells and their protease granzyme B with activation of airway epithelial cells. The key outcome is IL-25 production by epithelial cells. NK cell-induced epithelial IL-25 activates pulmonary group 2 innate lymphoid cells (ILC2s) and Th2 cells, leading to AAD.

\section{Results}

Maternal exposure to DEP enhances the type 2 immune response in offspring. Using mouse models, we previously showed that maternal exposure to DEP either before or during pregnancy predisposed offspring to $\operatorname{AAD}(13,14)$. For further studies, we chose the model with prepregnancy exposure. We favored it over its alternative with exposure during pregnancy because the former was more reflective of the human exposure that occurs randomly across 
A
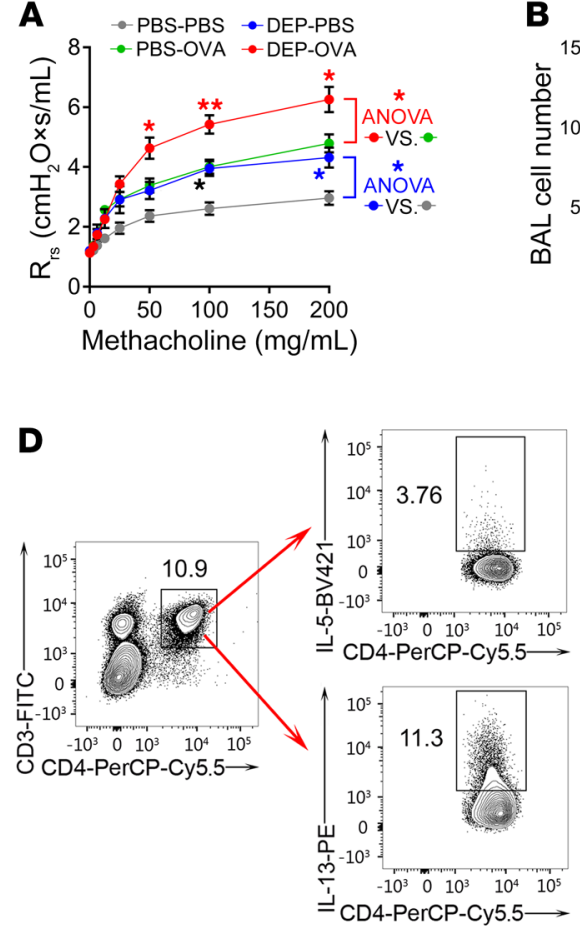

G
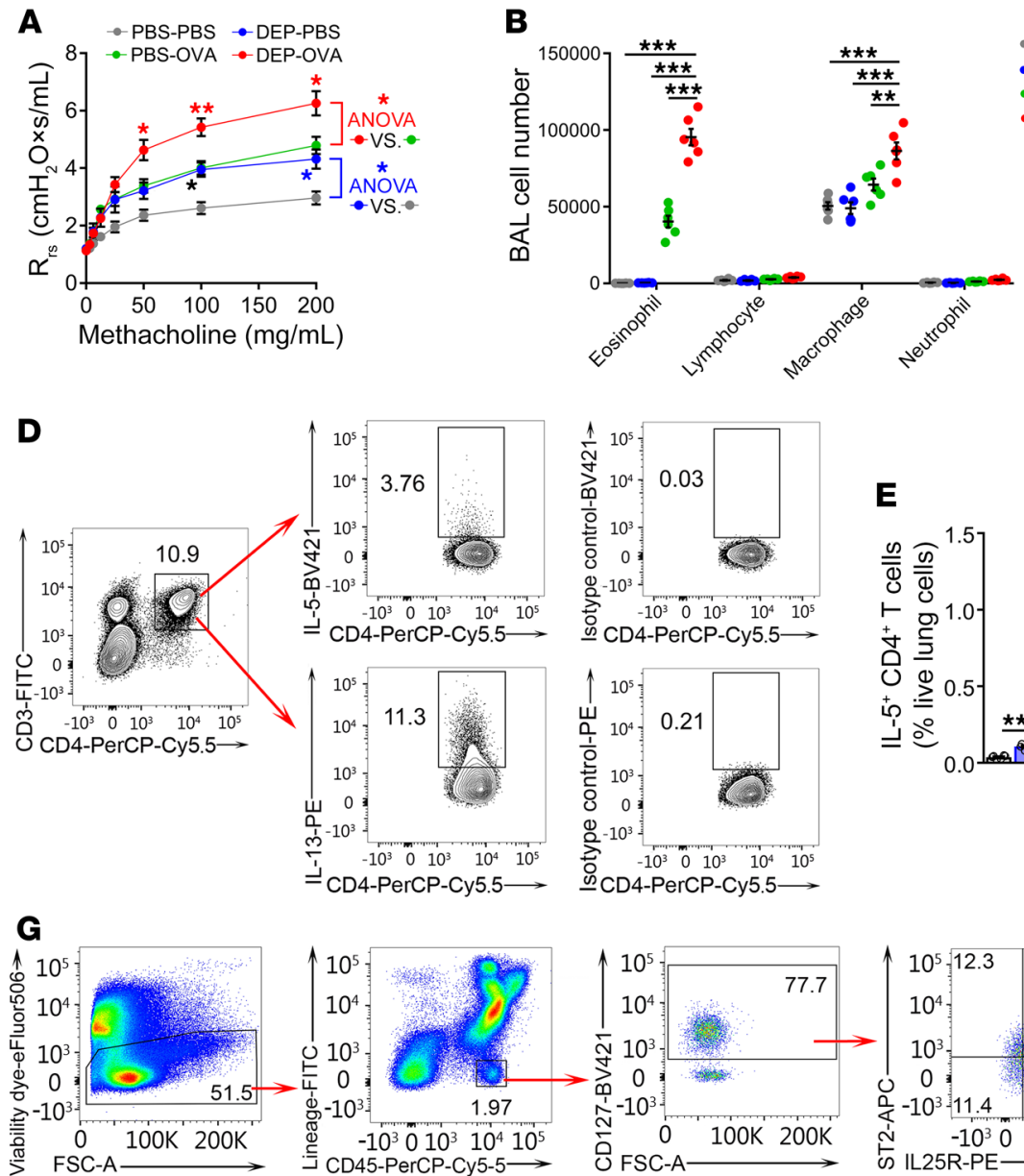

㝵 CD4-PerCP-Cy5.5 $\stackrel{-10^{3}}{\longrightarrow}$
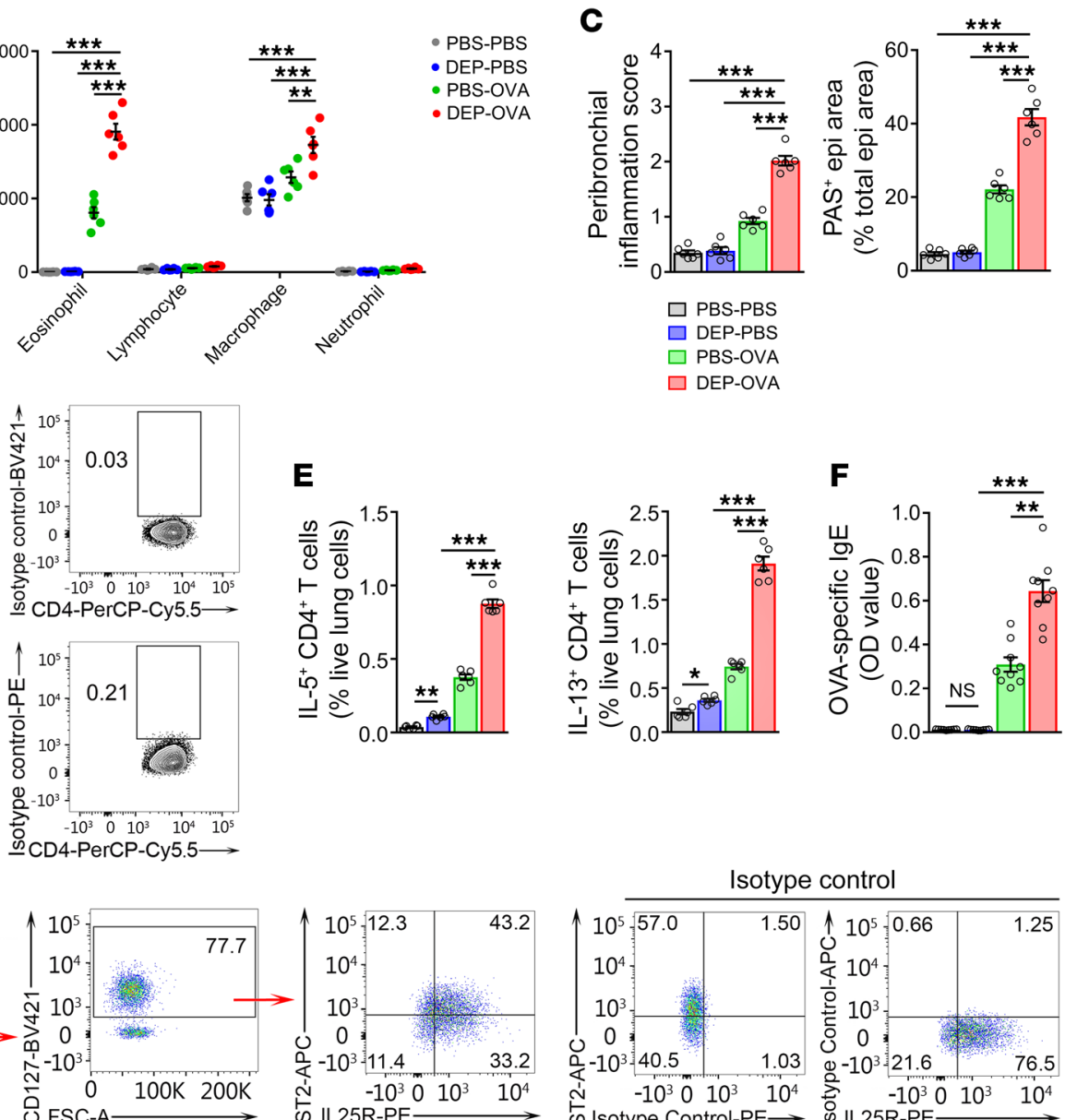

$\stackrel{\mathrm{N}}{0} \begin{array}{ccc}0 & 100 \mathrm{~K} & 200 \mathrm{~K} \\ \mathrm{O}\end{array}$

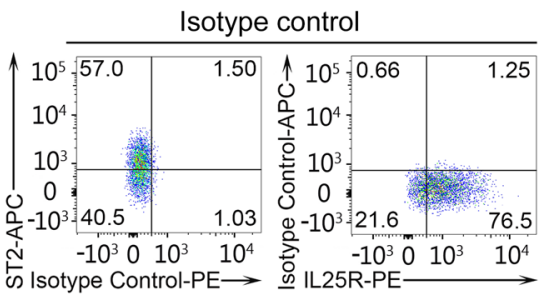

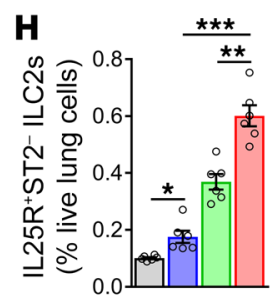
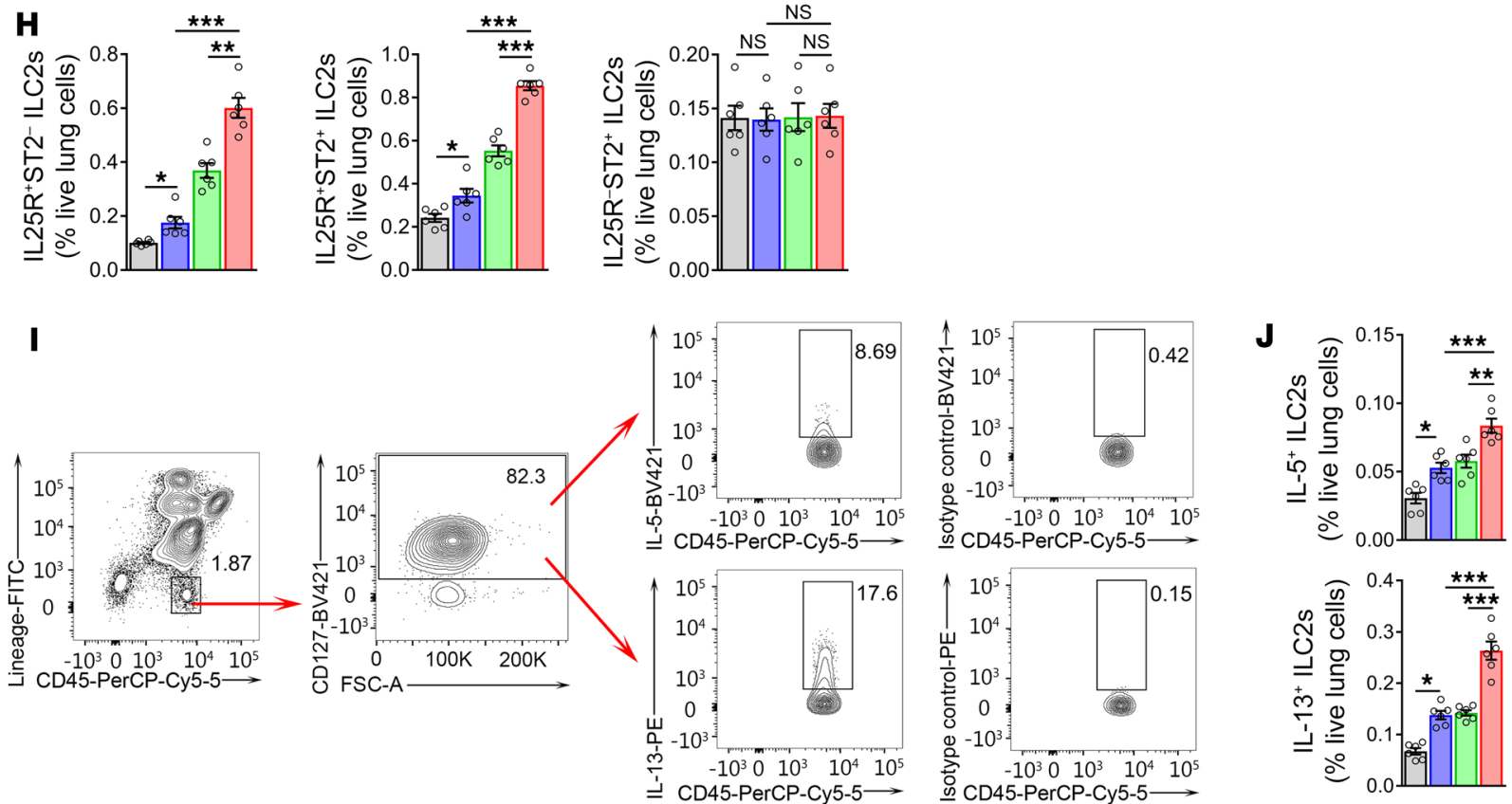
Figure 1. Maternal exposure to DEP enhances type $\mathbf{2}$ immune response and AAD in offspring. (A-J) AAD and type 2 immune response in lungs of PBS-PBS, DEP-PBS, PBS-OVA, and DEP-OVA pups. (A) Total lung resistance to methacholine in indicated groups of pups. $n=6$. Rrs, resistance of the respiratory system. (B) Leukocyte subsets in BAL fluid. $n=6$. (C) Peribronchial inflammation scores (left) and proportions of bronchial epithelial (epi) areas that are PAS (mucin)+ (right). $n=6$. (D) Flow cytometry (FC) plots to quantify pulmonary $\mathrm{IL}-5^{+}$and $\mathrm{IL}-13^{+} \mathrm{CD} 4^{+} \mathrm{T}$ cells. PMA/ionomycin-stimulated lung cell suspensions were stained for flow cytometry. After ex vivo stimulation with PMA/lonomycin, staining, exclusion of debris, doublets, and dead cells, live (eFluor506-) lung singlets were gated on $\mathrm{CD}^{+}{ }^{+} \mathrm{CD} 4^{+}$cells and then on cytokine ${ }^{+}$cells. (E) Percentages of cytokine ${ }^{+}$ CD4 ${ }^{+}$T cells in live lung cells. $n=6$. (F) OVA-specific IgE in serum. $n=9$. (C) FC plots to quantify lung ILC2 subsets. Live lung singlets (no ex vivo stimulation) were analyzed for Lineage/Lin markers (CD3, B220, CD11b, CD11c, Gr1, FceRl $\alpha$, and NK1.1) and CD45. CD45'Lin ${ }^{-}$cells were analyzed for CD127. CD127+ cells were analyzed for IL25R and ST2 to quantify IL25R+'ST2-, IL25R+'ST2+, and IL25R-ST2+ ILC2 subsets (CD45+Lin-CD127+

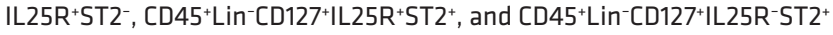
cells, respectively). (H) Percentages of ILC2 subsets in live lung cells. $n=6$. (I) Gating strategy to quantify pulmonary cytokine+ ILC2s. PMA/ionomycin-stimulated live singlets were gated on $\mathrm{CD}^{4} 5^{+} \mathrm{Lin}^{-}$cells, then on $\mathrm{CD} 127^{+}$ cells and finally on cytokine ${ }^{+}$cells. (J) Percentages of cytokine ${ }^{+}$ILC2s in live lung cells. $n=6$. Data are representative of 3 independent experiments and are shown as mean $\pm \mathrm{SEM} .{ }^{*} P<0.05 ;{ }^{* *} P<0.01$; ${ }^{* *} P<0.001,2$-way repeated-measures ANOVA with Bonferroni's post hoc test (A); 1-way ANOVA with Tukey's post hoc test (B, C, E, F, H, and J).

the lifetime with no particular connection with pregnancy. In our chosen model, C57BL/6 female mice received 6 i.n. applications of DEP or PBS before pregnancy (see Supplemental Figure $1 \mathrm{~A}$ for schematic illustration of the model; supplemental material available online with this article; https://doi.org/10.1172/ JCI130324DS1). Two weeks after the final application, females were mated with unexposed males. Pups were sensitized to OVA to initiate allergic response. Immunization included a single i.p. injection of low-dose OVA in alum on day 5 after birth. Separate sets of pups were injected with PBS. To elicit AAD, immunized pups were i.n. challenged with OVA on days 23,24 , and 25 . Pups receiving PBS on day 5 were challenged with PBS. All pups were analyzed on day 28 after birth. The following groups of pups were studied: pups born to DEP mothers and exposed to PBS (DEPPBS pups) or OVA (DEP-OVA pups) after birth; and pups born to PBS mothers and exposed to PBS (PBS-PBS pups) or OVA (PBSOVA pups) after birth. In PBS-OVA pups, AAD features (airway hyperresponsiveness [AHR] to methacholine, eosinophils in the bronchoalveolar lavage (BAL) fluid, peribronchial inflammation, and goblet cell hyperplasia; ref. 13 and Figure 1, A-C) were mild, reflecting immaturity of the neonatal immune system. Preconceptional DEP greatly exacerbated these features, leading to fully manifested AAD (ref. 13 and Figure $1, A-C$ ). To begin to address mechanisms of AAD intensification, we analyzed the type 2 immune response in the lungs (Figure 1, D-J). We found that among all studied groups of pups, DEP-OVA pups had the highest frequencies of IL- $5^{+}$and IL- $13^{+} \mathrm{CD} 4^{+} \mathrm{T}$ cells in their lungs (Figure 1, D and E) and the highest levels of OVA-specific IgE in the serum (Figure 1F). DEP-OVA pups also had the highest frequencies of pulmonary IL25R ${ }^{+}$ILC2s (CD $45^{+} \mathrm{Lin}^{-} \mathrm{CD} 127^{+} \mathrm{IL} 25 \mathrm{R}^{+} \mathrm{ST} 2$ cells and $\mathrm{CD}^{2} 5^{+} \mathrm{Lin}^{-} \mathrm{CD} 127^{+} \mathrm{IL} 25 \mathrm{R}^{+} \mathrm{ST} 2^{+}$cells; Figure 1, G and H) and the highest frequencies of pulmonary IL- $5^{+}$and IL-13+ ILC2s $\left(\mathrm{CD} 45^{+} \mathrm{Lin}^{-} \mathrm{CD} 127^{+} \mathrm{IL}-5^{+}\right.$cells and $\mathrm{CD} 45^{+} \mathrm{Lin}^{-} \mathrm{CD} 127^{+} \mathrm{IL}-13^{+}$cells; Figure 1, I and J). IL25R ${ }^{+}$ILC2s and Th2 cells were also modestly increased in DEP-PBS pups (Figure 1, E, H, and J). IL25R- ILC2s $\left(\mathrm{CD}^{2} 5^{+} \mathrm{Lin}^{-} \mathrm{CD} 127^{+} \mathrm{IL}^{25 \mathrm{R}^{-} \mathrm{ST}} 2^{+}\right.$cells) were not affected by maternal exposure to DEP (Figure 1, G and H). Of note, our CD45/Lin/ CD127/IL25R/ST2-based gating strategy accurately captured all ILC2s. CD $45^{+} \mathrm{Lin}^{-} \mathrm{CD} 127^{+} \mathrm{IL} 25 \mathrm{R}^{+} \mathrm{ST} 2^{-}$cells, $\mathrm{CD} 45^{+} \mathrm{Lin}^{-} \mathrm{CD} 127^{+}$

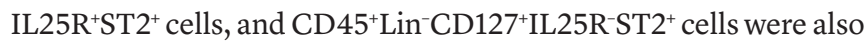
positive for other ILC2 markers, such as GATA3, CD25, and ICOS; $\mathrm{CD}^{4} 5^{+} \mathrm{Lin}^{-} \mathrm{CD} 127^{+}$IL25R $-\mathrm{ST} 2{ }^{-}$cells were negative for or expressed very low levels of these markers (Supplemental Figure 1B).

Maternal signals prime offspring NK cells. ILC2s are not the only ILC subset in the lung. The most dominant subset, accounting for $10 \%$ of pulmonary lymphocytes, are NK cells. We asked whether the maternal influence extends to this ILC subset as well. DEP-OVA pups had more NK cells in the lung than PBS-OVA pups had (Figure 2A and Supplemental Figure 2A). Compared with pulmonary NK cells from PBS-PBS and PBS-OVA pups, pulmonary NK cells from DEP-PBS and DEP-OVA pups were more mature, as illustrated by lower frequency of the $\mathrm{CD} 11 \mathrm{~b}^{-} \mathrm{CD} 27^{+}$

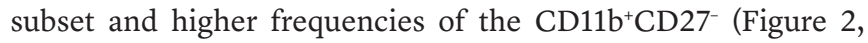
B and C, and Supplemental Figure 2A), CD11 ${ }^{+}$CD27-KLRG1 ${ }^{+}$, and $\mathrm{CD} 11 \mathrm{~b}^{+} \mathrm{CD} 27^{-} \mathrm{KLRG1}^{+}{ }^{+} \mathrm{LCC}^{+}$(Figure 2D and Supplemental Figure 2A) subsets. Downregulation of CD27 and induction of CD11b, KLRG1, and Ly6C are indicative of terminal maturation and development of NK cell memory and predictive of enhanced effector functions $(15,16)$. Indeed, pulmonary NK cells from pups of DEP-exposed mothers showed increased degranulation, denoting increased secretion of granule proteins, including granzymes (DEP-OVA pups; Figure 2E), and enhanced capacity to produce IL-5 and IL-13 (DEP-PBS and DEP-OVA pups; Figure $2 F)$. This finding was intriguing because NK cells are not generally thought to secrete type 2 cytokines; instead, they are the major source of type 1 cytokines, such as IFN- $\gamma$. Particularly interesting was production of IL- 5 and IL-13 by DEP-PBS NK cells. The result suggested that DEP-linked signals have the capacity to induce the type 2 differentiation program in NK cells. In support of this hypothesis, DEP-PBS NK cells showed increased expression of the transcriptional master regulator of the type 2 polarization program, GATA3 (Figure 2G). Collectively, our data indicated that maternal exposure to DEP imprinted the proallergic maturation program in offspring NK cells.

$N K$ cells drive the type 2 immune response and $A A D$. We were intrigued by NK cell activation in our model because NK cells are not traditionally believed to participate in asthma/AAD. We decided to investigate this potential link further. To study the importance of NK cells in type 2 response and AAD, we took 3 approaches. In the first approach, we used the $N c r 1^{i c r e} R 26^{\text {DTA }}$ model of NK cell deficiency (17). The Ncri ${ }^{i c r e}$ knockin allele has the YFP-IRES-Cre cassette inserted into the 3' UTR region of the Nor1 gene (encoding the NKp46 protein), enabling coordinated transcription of Cre and endogenous Ncr1. Ncrl is one of the most specific genes of the NK cell lineage $(17,18)$. In our model, $91.36 \%$ $\pm 1.42 \%$ of lung NKp $46^{+}$cells expressed NK1.1 and lacked CD3, fitting into the traditional definition of NK cells (Supplemental Figure 2B). Thus, our results confirmed previous reports of a high 
degree of coexpression between NKp46 and other markers of NK

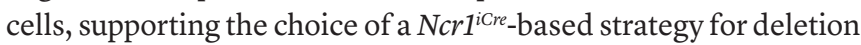
of NK cells. The other element of the NK cell-deletion strategy is the $R 26^{\text {DTA }}$ allele. This allele has the diphtheria toxin A (DTA) gene and the upstream loxP-flanked STOP codon inserted into the ubiquitously expressed ROSA26 locus. The STOP codon prevents DTA transcription. A cross of $N c r 1^{i \text { ire }}$ to $R 26^{\text {DTA }}$ mice enables excision of STOP by Cre and transcription of DTA in NKp $46^{+}$cells. As a result, $\mathrm{NKp} 46^{+}$cells are killed, leaving other cells intact. In our experiment, DEP-exposed $\mathrm{Ncr}^{\mathrm{iCr} /+}$ mothers were mated with unexposed $R 26^{\text {DTA/DTA }}$ fathers to generate NK cell-deficient $\mathrm{Ncr}^{i \mathrm{irr} /+}$ $R 26^{\text {DTA/+ }}$ and NK cell-sufficient $R 26^{\text {DTA } /+}$ littermates. All pups were immunized and challenged with OVA. $N c r 1^{\text {iCre/+}} R 26^{\text {DTA/+ }}$ pups lacked NK cells, but had normal frequencies of NKT cells and conventional $\mathrm{CD}^{+}{ }^{+} \mathrm{NK} 1.1^{-} \mathrm{T}$ cells (Supplemental Figure 2, C and D). NK cell deficiency led to marked reduction in pulmonary IL- 5 and IL-13 (Figure 3, A and B), IL25R ${ }^{+}$and type 2 cytokine ${ }^{+}$ILC2s (Figure $3, \mathrm{E}$ and $\mathrm{F}$ ), $\mathrm{IL}^{-} 5^{+}$and $\mathrm{IL}-13^{+} \mathrm{CD} 4^{+} \mathrm{T}$ cells (Figure $3 \mathrm{C}$ ), OVA-specific $\operatorname{IgE}$ in the serum (Figure 3D), and all features of AAD, including AHR to methacholine (Figure $3 \mathrm{G}$ and Supplemental Figure 2E), eosinophils (Figure 3I and Supplemental Figure 2F), peribronchial inflammation (Figure 3H), and goblet cell hyperplasia (Figure 3J).

In addition to being expressed by NK cells, NKp46 is also detected on a few rare lymphocyte subsets, such as ILC1s and NKp46 ILC3s, and a minute fraction of NKT cells. Consistent with these findings, $\mathrm{Ncr}^{\mathrm{iCre} /{ }^{+}} \mathrm{R} 26^{\mathrm{DTA} /+}$ mice lack ILC1s and NKp $46^{+}$ILC3s (19). To ensure that the observed effects of NKp $46^{+}$cells on AAD represented effects of conventional NK cells and not the effects of NKp46+ ILC1/ILC3/NKT cells, we performed 2 experiments. Conventional NK cells can be distinguished from ILC1/ILC3s and NKT cells by expression of CD127 and CD3 by ILC1/ILC3s and NKT cells, respectively. Building on this, to exclude the roles of $\mathrm{NKp} 46^{+}$ILCs and NKT cells, we reconstituted $\mathrm{Ncr} 1^{\text {iCre/+}} \mathrm{R} 26^{\text {DTA/+ }}$ pups with $\mathrm{CD}_{127^{-}} \mathrm{NK}$ cells (see Supplemental Figure 3B for diagram of the experimental strategy). To purify CD127- NK cells, we used the negative selection-based Miltenyi NK Cell Isolation Kit, which included anti-CD3 and which we additionally supplemented with anti-CD127. The purified cell population was devoid of NKT cells $\left(\mathrm{NK} 1.1^{+} \mathrm{CD}^{+}\right)$, ILC1s $\left(\mathrm{CD} 45^{+} \mathrm{Lin}^{-} \mathrm{CD} 127^{+}\right.$Eomes T-bet ${ }^{+} \mathrm{NKp} 46^{+}$), NKp46 $6^{+}$and NKp46- ILC3s (CD45 ${ }^{+} \mathrm{Lin}^{-} \mathrm{CD} 127^{+}$ $\mathrm{ROR} \gamma \mathrm{t}^{+} \mathrm{NKp} 46^{+}$and $\left.\mathrm{CD}^{+} 5^{+} \mathrm{Lin}^{-} \mathrm{CD} 127^{+} \mathrm{ROR} \gamma \mathrm{t}^{+} \mathrm{NKp} 46^{-}\right)$, and ILC2s $\left(\mathrm{CD}^{4} 5^{+} \mathrm{Lin}^{-} \mathrm{CD} 127^{+} \mathrm{NKp} 46^{-} \mathrm{GATA}^{+}\right.$) (Supplemental Figure 3A). Transfer of CD127 NK cells from NK cell-sufficient DEPOVA pups into NK cell-deficient $\mathrm{Ncrl}^{\mathrm{iCre} /+} \mathrm{R} 26^{\mathrm{DTA} /+}$ DEP-OVA pups led to complete reconstitution of IL25R $\mathrm{R}^{+}$ILC2s (Figure 3K) and features of AAD (Figure 3, L-N), supporting our conclusions regarding the dependence of these traits on conventional NK cells and underscoring the lack of meaningful involvement of $\mathrm{NKp} 46^{+}$ ILC1/ILC3s and NKT cells.

Finally, we used the anti-asialo-GM1 antiserum to deplete NK cells (diagram of the experimental strategy in Supplemental Figure 3C). Anti-asialo-GM1 does not delete ILC1/ILC3s and NKT cells (refs. 20, 21 and Supplemental Figure 3D). Pups of DEP-exposed mothers were injected with the anti-asialo-GM1 antiserum 1 day before immunization, 1 day before challenge, and on the last day of challenge. Anti-asialo-GM1 reduced all features of AAD (Figure 3, O-Q).
Type 2 immune response and $A A D$ are dependent on $I L-25$. We previously showed that airway inflammation and AAD in our model were dependent on IL-1 $\beta$ and its downstream target IL-17A, which mediates AHR (13). IL-1 $\beta$ is a recognized enhancer of the type 2 immune response, activating both ILC2s and Th2 cells. We therefore wondered whether IL-1 $\beta$ and NK cells are linked. $N c r 1^{i \mathrm{Cr} /+}$ $R 26^{\text {DTA/+ }}$ and $R 26^{\text {DTA/+ }}$ DEP-OVA littermates did not differ in regard to levels of mRNA for IL-1 $\beta$ and IL-17A, indicating that NK cells do not control these cytokines (Supplemental Figure 4A). We also tested to determine whether the opposite is true, i.e., whether IL-1 $\beta$ regulates NK cells. To this end, DEP-OVA pups were injected with a neutralizing anti-IL-1 $\beta$ antibody (or control IgG) on postnatal day 5 (1 day before immunization) and then on postnatal day 22 (1 day before the first challenge with OVA; diagram of experimental strategy in Supplemental Figure 4B). Although this treatment depleted IL-1 $\beta$ (Supplemental Figure 4C) and prevented development of AAD in our previous study (13), it had no effect on NK cell maturation (Supplemental Figure 4, D and E) and degranulation (Supplemental Figure 4F) and type 2 cytokine production (Supplemental Figure 4G). Taken together, our data indicate that IL-1 $\beta$ and NK cells are not linked and therefore regulate type 2 inflammation and $\mathrm{AAD}$ through independent routes.

Having established that NK cells were unlikely to activate ILC2s and Th2 cells through IL-1 $\beta$ (Supplemental Figure 4A), we shifted our focus to more traditional regulators of these cells, i.e., epithelial cytokines IL-25, IL-33, and thymic stromal lymphopoietin (TSLP). At 72 hours after challenge with OVA, DEP-OVA pups overexpressed and oversecreted IL-25 (Figure 4, A and B) and expressed and secreted normal levels of IL-33 and TSLP (Figure $4, \mathrm{~A}$ and B) in their lungs. We reasoned that IL-25 induction could explain expansion of IL-25R $\mathrm{R}^{+}$ILC2s. To examine the importance of IL-25 in IL25R+ ILC2 activation, other features of the type 2 immune response, and AAD, DEP-OVA pups were injected with an IL-25-depleting antibody 1 day before immunization and then 1 day before the first challenge with OVA (see Supplemental Figure 4B for diagram of the experimental strategy). The anti-IL-25 reduced pulmonary IL25R $\mathrm{R}^{+}$ILC2s (Figure 4C), OVA-specific IgE in the serum (Figure 4D), and features of AAD (Figure 4, E-H, and Supplemental Figure 4, $\mathrm{H}$ and I) to levels comparable to those in PBS-OVA pups. Of note, IL-25 depletion had no effect on IL-1 $\beta$ and IL-17A (Supplemental Figure 4J). In conclusion, IL-25 was an important driver of DEP-programmed, allergen-elicited type 2 inflammation and AAD.

Normal levels of IL-33 and TSLP mRNAs and proteins were somewhat surprising, given the importance of these cytokines for lung inflammation in other models of AAD. To obtain definitive answers regarding the contributions of IL-33 and TSLP to AAD in our model, we individually depleted these cytokines in DEP-OVA pups using specific antibodies (diagram of experimental strategy in Supplemental Figure 4B). Separate groups of pups received control IgGs. Injections of anti-IL-33 and anti-TSLP antibodies led to substantial reductions of targeted cytokines (Figure 4, I and M), but had no effect on AAD (Figure 4, J-L and N-P), indicating that IL-33 and TSLP were redundant in our model. These results were consistent with lack of DEP effects on IL-25R-ST2 ${ }^{+}$ILC2s (Figure 1H).

$N K$ cells are upstream of IL-25. We asked whether IL-25 and NK cells are linked. To study the effect of NK cells on IL-25, we used NK cell-deficient pups. NK cell deficiency led to marked 
A

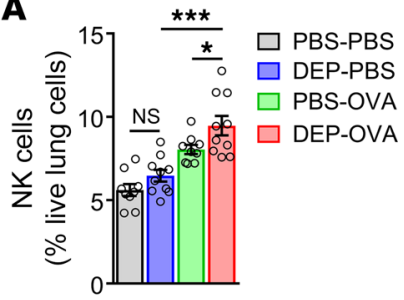

B

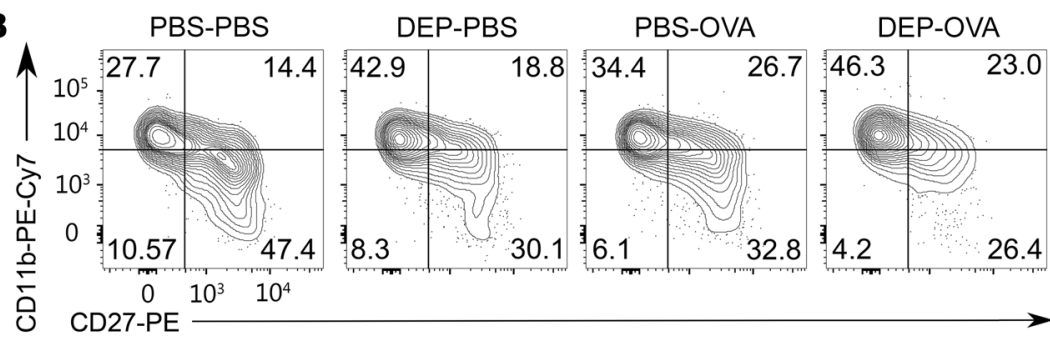

C

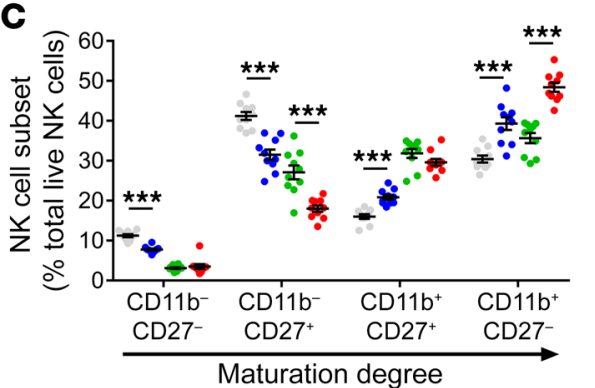

D +

PBS-PBS

- DEP-PBS

- PBS-OVA

- DEP-OVA
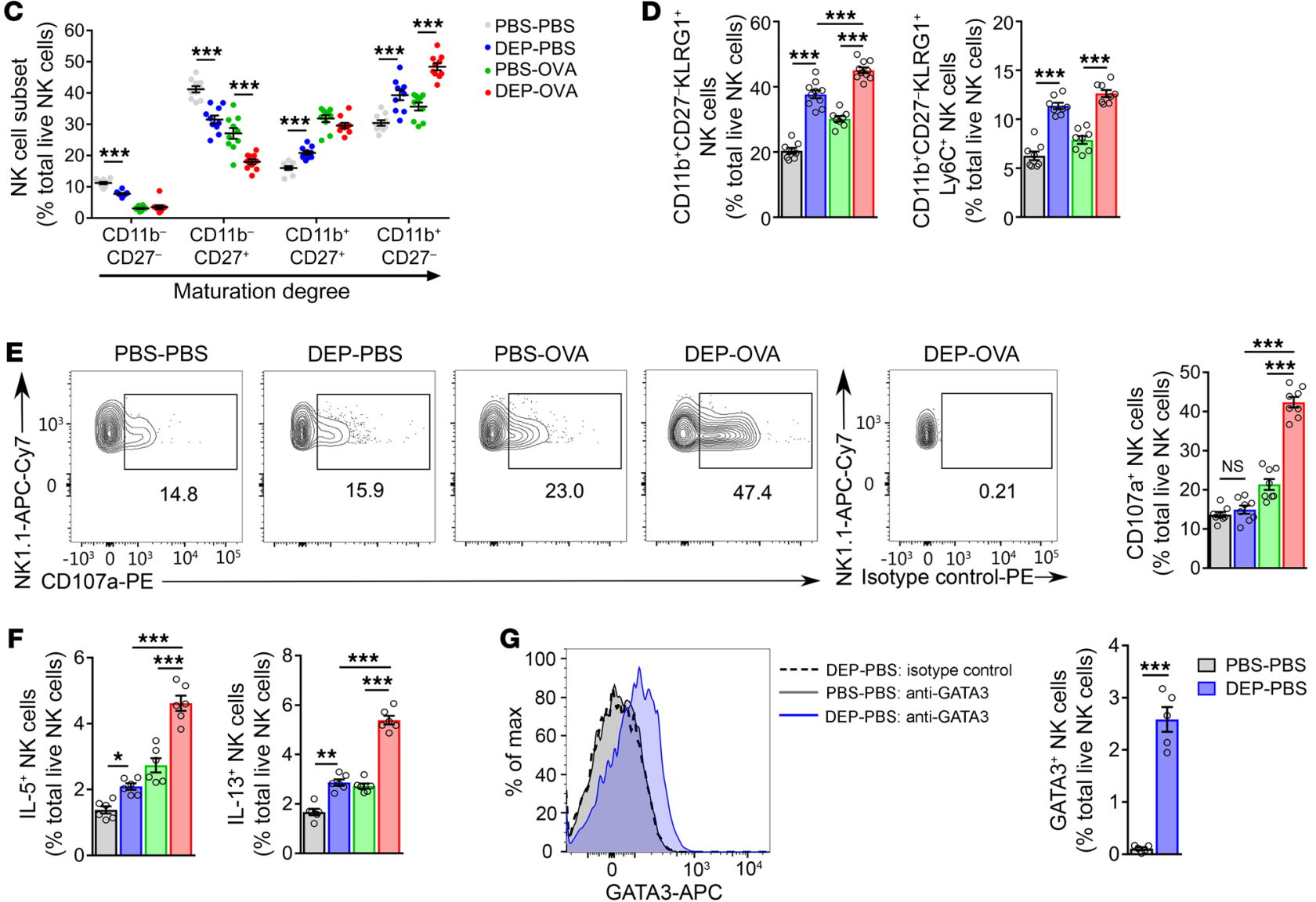

Figure 2. DEP NK cells have increased capacity to produce type $\mathbf{2}$ cytokines and degranulate. (A-C) Frequencies and features of lung NK cells in PBS-PBS, DEP-PBS, PBS-OVA, and DEP-OVA pups. (A) Percentages of NK cells (CD3-CD19-NK1.1+) in live lung cells. $n=9-10$ mice per group. (B) Flow cytometry plots to detect NK cell subsets. CD3-CD19-NK1.1+ live lung cells were analyzed for CD11b and CD27. (C and D) Percentages of indicated subsets in live lung NK cells. $n=9-10$ mice per group (B-D: gating strategy in Supplemental Figure 2A). (E) Left: flow cytometry plots to measure degranulated (CD107a ${ }^{+}$) NK cells in lung digests. Lung cells were incubated at $37^{\circ} \mathrm{C}$ with PE-labeled anti-CD107a or isotype control IgG, monensin, brefeldin A, IL-2, and IL-15, and then stained with eFluor506 and antibodies for surface markers. Live NK cells (eFluor506-CD3-CD19-NK1.1+) were analyzed for NK1.1 versus CD107a. Right: percentages of CD107a+ NK cells in live lung NK cells. $n=8$. (F) Percentages of IL-5+ and IL-13+ NK cells in live lung NK cells. Result was obtained after ex vivo stimulation with PMA/ionomycin. $n=6$. (G) Left: flow cytometry plot to identify GATA3 ${ }^{+}$NK cells. CD3-CD19-CD127-NK1.1 live lung cells (no ex vivo stimulation) from PBS-PBS and DEP-PBS pups were analyzed for GATA3 or binding of an isotype control immunoglobulin. Right: percentages of GATA3 ${ }^{+}$NK cells in live lung NK cells. $n=5$. Data are representative of 2 independent experiments and are shown as mean \pm SEM. ${ }^{*} P<0.05 ;{ }^{* *} P<0.01 ;{ }^{* * *} P<0.001,1$-way ANOVA with Tukey's post hoc test (A and C-F); 2-tailed unpaired $t$ test (G).

reduction of pulmonary IL-25 mRNA and protein (Figure 5, A-C; and Supplemental Table 1). The loss of IL-25 was most evident in airway epithelial cells (Figure 5C). These cells were the principal sources of IL-25 in control pups. NK cell deficiency did not affect IL-33 and TSLP, suggesting relative selectivity of NK cells for IL-25 (Figure 5, A and B).

We then wanted to determine whether or not IL-25 induction was the key mechanism responsible for induction of the type 2 immune response and AAD by NK cells. To test this theory, Ncr1 ${ }^{\text {icre/ }}+$ $R 26^{\text {DTA/+ }}$ pups of DEP-exposed mothers were administered with recombinant IL-25 during the immunization phase (on postnatal days 5 and 6) and then during the challenge phase (on postnatal days 23, 24, and 25) (diagram of the experimental strategy in Supplemental Figure 5). IL-25 reconstitution led to restoration of pulmonary IL25R $\mathrm{R}^{+}$ILC2s (Figure 5D) and AAD (Figure 5, E-G), indicating that IL-25 induction was critical for NK cell regulation 

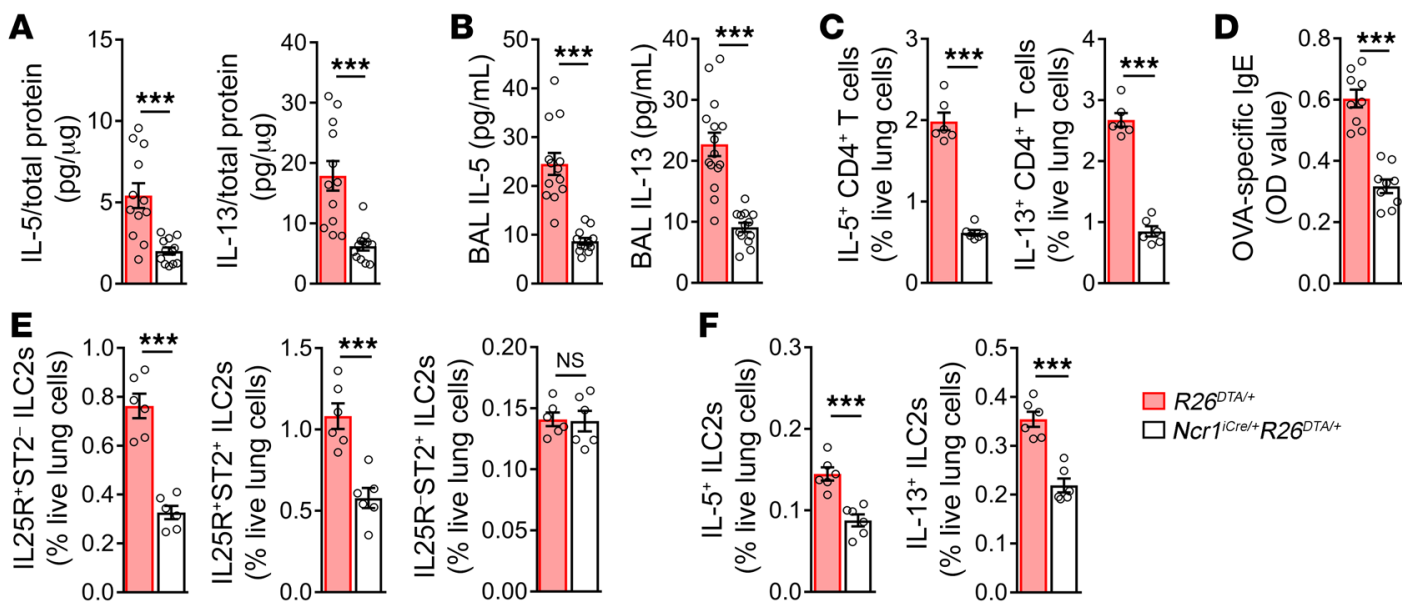

G

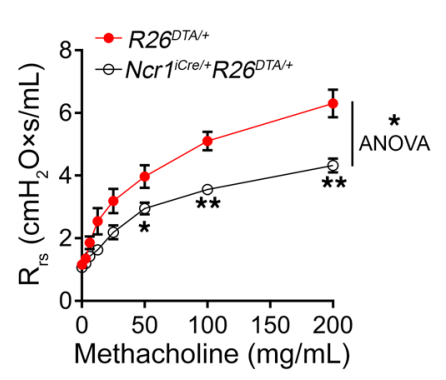

H
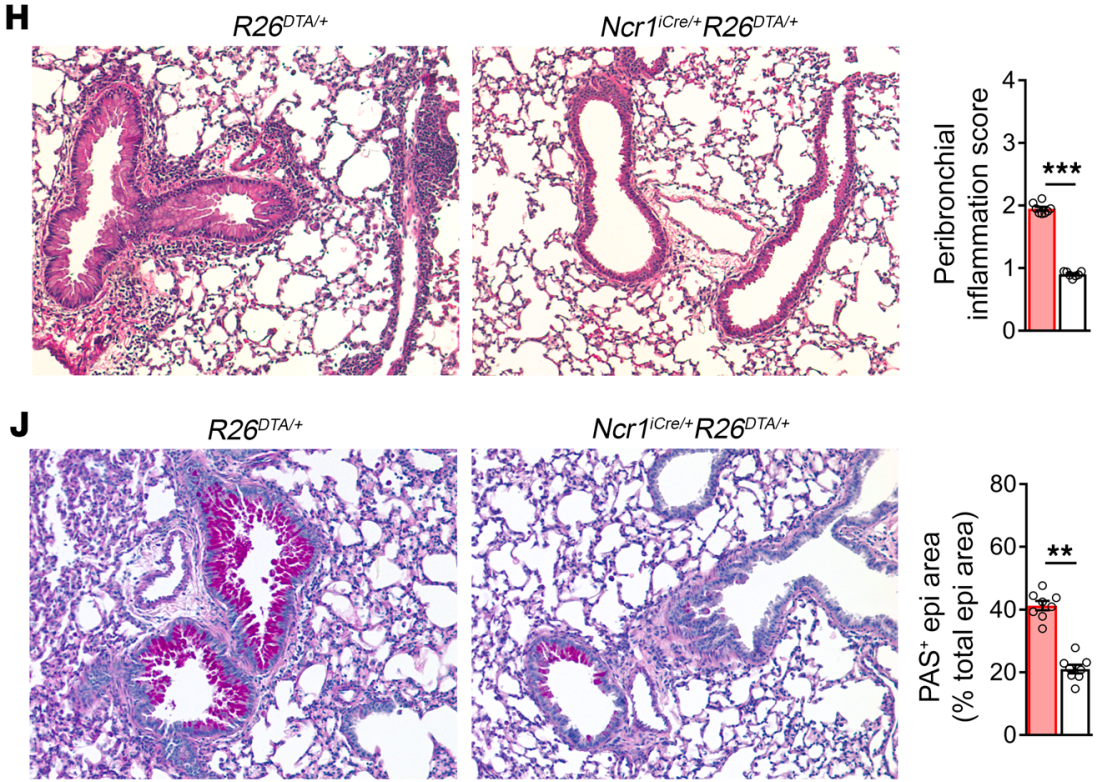

$\mathbf{K}$
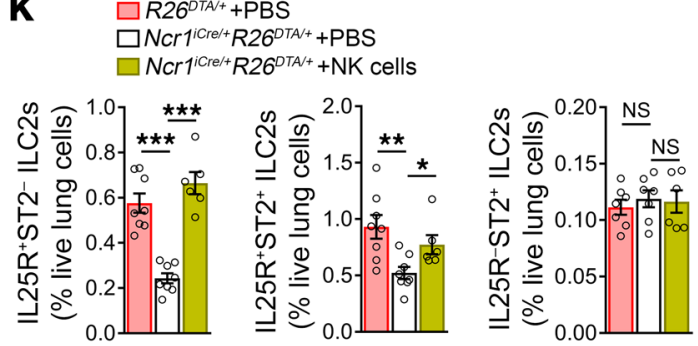

$\mathbf{L}$

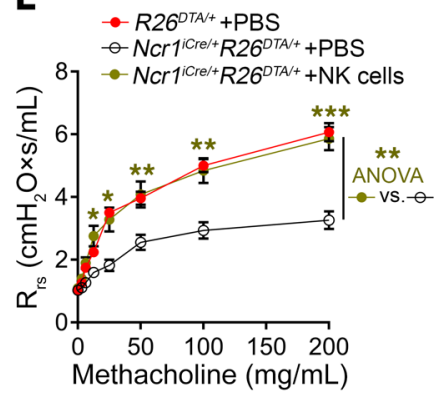

M

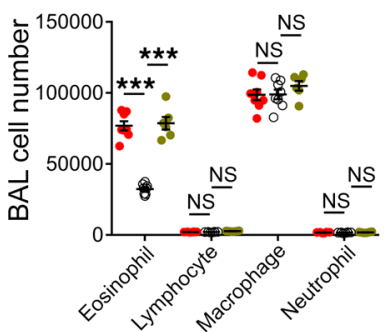

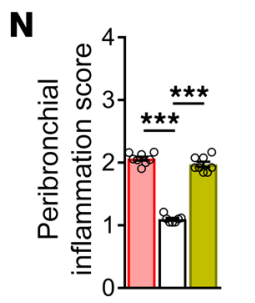
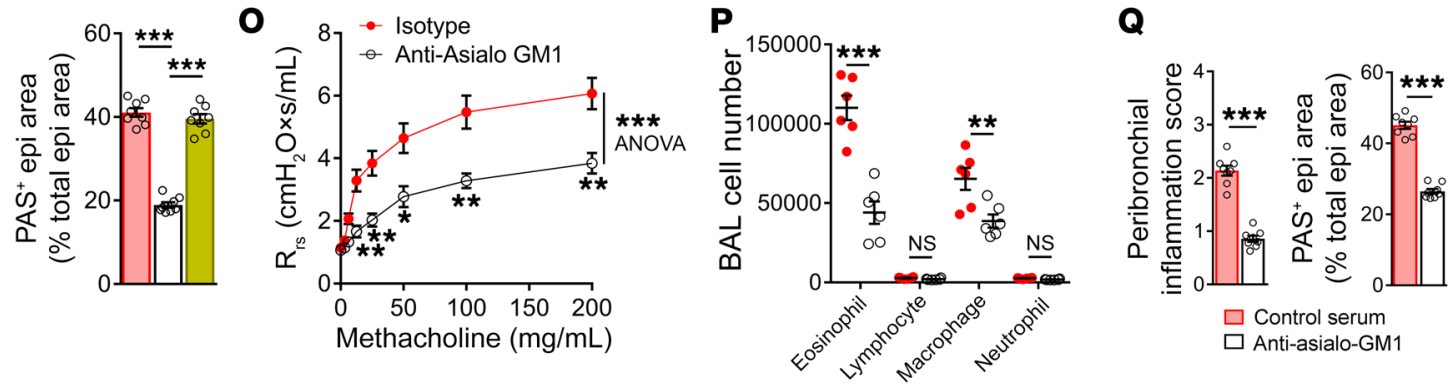
Figure 3. The type $\mathbf{2}$ immune response and AAD are driven by NK cells. The type 2 immune response and AAD in $\mathrm{NCr}^{i \mathrm{Cr} /+} \mathrm{R}^{2} 6^{\mathrm{DTA} /+}$ and $R 26^{\mathrm{DTA} /+} \mathrm{DEP}-$ OVA pups (A-J), Ncr $7^{i \text { Cre/+}} R 26^{\text {DTA } /+}$ and $R 26^{\text {DTA } /+}$ DEP-OVA pups after injection

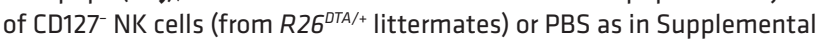
Figure 3B (K-N), and WT DEP-OVA pups after injection of anti-asialo-GM1 or control sera as in Supplemental Figure 3C (0-Q). (A and B) Cytokines in lung homogenates (A, $n=12$ ) and BAL fluid (B, $n=13-15)$. (C, E, F, and $\mathbf{K}$ ) Cytokine ${ }^{+} \mathrm{CD}^{+}{ }^{+}$T cells $(\mathbf{C}, n=6), \mathrm{IL} 25 \mathrm{R}^{+} \mathrm{ST2} 2^{-}, \mathrm{IL} 25 \mathrm{R}^{+} \mathrm{ST} 2^{+}$, and IL25R-ST2 ILC2s (E, $n=6 ; \mathbf{K}, n=6-8)$ and cytokine ${ }^{+}$LLC2s $_{(\mathbf{F}, n=6)}$ in live lung cells. (D) OVA-specific IgE in serum. $n=9$. (G, $\mathbf{L}$, and $\mathbf{O})$ Total lung resistance to methacholine. $n=6$ (G and $\mathbf{L}) ; n=5(\mathbf{O})$. (H, J, N, and Q) H\&E- and PASstained lung sections, inflammation scores, and areas of PAS ${ }^{+}$epithelium. Original magnification, $\times 100 . n=7(\mathbf{H}) ; n=8(\mathbf{J}, \mathbf{N}$, and $\mathbf{Q})$. (I, M, and $\mathbf{P})$ Leukocyte subsets in the BAL fluid. $n=8(\mathbf{I}) ; n=6-8(\mathbf{M})$; and $n=6$ (P). Data are representative of $\mathbf{2}(\mathbf{A}$ and $\mathbf{B}$ ) or $\mathbf{3}(\mathbf{C}-\mathbf{Q})$ independent experiments and are shown as mean \pm SEM. ${ }^{*} P<0.05 ;{ }^{* *} P<0.01 ;{ }^{* * *} P<0.001$, 2-tailed unpaired $t$ test for (A-F, H-J, $\mathbf{P}$, and $\mathbf{Q})$ ) 1-way ANOVA with Tukey's post hoc test (K, $\mathbf{M}$, and $\mathbf{N})$; 2-way repeated-measures ANOVA with Bonferroni's post hoc test $(\mathbf{G}, \mathbf{L}, \mathbf{0})$.

of these traits. Therefore, NK cells promoted the type 2 response and AAD via IL-25.

NK cells serve as carriers of susceptibility to AAD. To test whether NK cells are carriers of AAD predisposition in our model, we transferred $\mathrm{CD}_{127^{-}} \mathrm{NK}$ cells from WT pups of DEP- and PBSexposed mothers (referred to as DEP NK cells and PBS NK cells, respectively) into normal (no DEP) age-matched recipients (diagram of the experimental strategy in Supplemental Figure 6; see protocol 1). The third group of recipients was injected with PBS without cells. All recipients were born to unexposed mothers and had the $\mathrm{Ncr}^{\mathrm{iCre} /+} R 26^{\mathrm{DTA} /+}$ (NK cell ${ }^{\text {null }}$ ) genotype. Intratracheal (i.t.) transfer took place at 3 weeks of age, after neonatal (postnatal day 5) immunization of recipients. Donors were not immunized. Following NK cell transfer/PBS injection, recipients were challenged with OVA. PBS-injected recipients had a low level of IL-25 in the BAL fluid (Figure 6A), low numbers of IL25R $\mathrm{R}^{+}$ILC2s (Figure 6B), and minimal AAD (Figure 6, C and D), reflecting immaturity of the immune system at the time of immunization. Transfer of PBS NK cells did not have any effects on these traits. In contrast, transfer of DEP NK cells led to increased production of IL-25, expansion of IL25R+ ILC2s, and exacerbation of AAD. From this result, we concluded that DEP NK cells were programmed to promote the type 2 immune response and AAD and that this program made them sufficient to transmit AAD susceptibility to normal recipients. We then designed an experiment to determine whether the DEP-linked program persisted in the NK cell lineage beyond early postnatal life into adulthood using adult (6 weeks old) offspring of DEP-exposed and PBS-exposed mothers as NK cell donors (Supplemental Figure 6, protocol 2). The overall experimental design was the same as that used for 3-week-old donors - NK cells (or PBS) were i.t. transferred into immunized, age-matched (6 weeks old), NK cell-deficient recipients. Immunization (a single i.p. dose of OVA in alum) took place 17 days before NK cell/PBS injection. One day following NK cell/PBS injection, recipients underwent their first challenge with OVA. Compared with recipients of PBS NK cells and recipients of PBS, recipients of DEP NK cells had increased IL-25 in the BAL fluid (Figure 6E) and increased numbers of pulmonary IL25R $\mathrm{R}^{+}$ILC2s (Figure $6 \mathrm{~F}$ ) and showed exac- erbation of AAD (Figure 6, G and $\mathrm{H}$ ). This result indicated persistence of NK cell programming into adulthood. In addition, the result indicated that, after achieving maturity and transitioning into adulthood, the lung remained amenable to signals emitted by DEP NK cells, continuing to respond by mounting type 2 inflammation and AAD.

$N K$ cell dominance is unique to maternal DEP-programmed AAD. We then explored whether or not NK cell participation was a general feature of AAD. To this end, we performed 2 experiments. In the first experiment, we used normal (no maternal DEP) adult mice and the conventional OVA-based model of AAD (diagram of the experimental strategy in Supplemental Figure 7). Experimental mice were adult offspring of unexposed $\mathrm{Ncr}^{\mathrm{icre} /+}$ mothers and

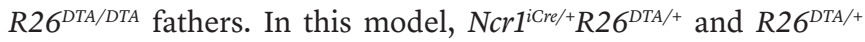
adult littermates had similar numbers of lung ILC2s (Figure 7A) and the same magnitude of AAD (Figure 7, B-E). Our results agreed with a recently published study that also used $N c r 1^{\text {icre }} R 26^{\text {DTA }}$ mice to show a lack of any meaningful involvement of NK cells in adult OVA- and house dust mite extract-based (HDM-based) models of AAD (22). Taken together, our data showed that NK cells are redundant for allergen-driven, DEP-free AAD in adult mice. We then examined NK cell engagement in an early life model of allergen-driven AAD. We addressed this by subjecting $\mathrm{Ncr}^{i \mathrm{ire} /+}$ mothers and their $\mathrm{Ncr}^{\mathrm{iCre} /+} R 26^{\mathrm{DTA} /+}$ and $R 26^{\mathrm{DTA} /+}$ neonates to our PBSOVA exposure scheme (Supplemental Figure 1A). As discussed earlier, in PBS-OVA pups, due to immaturity of their immune system, immune responses are of low magnitude; nevertheless, these pups develop low-grade airway eosinophilia and AHR that clearly differentiate them from PBS-PBS pups (13). As was the case with the adult model, numbers of ILC2s (Figure 7F) and eosinophils (Figure $7 \mathrm{H}$ ), AHR (Figure 7G), and other AAD features (Figure 7I) in PBS-OVA Ncr ${ }^{i \mathrm{Cre} /}+R 26^{\text {DTA/+ }}$ pups were the same as those in PBS-OVA R26 $6^{\text {DTA } /+}$ pups. By comparing results of this experiment with data in Figure 3, G-J, we also noted that AAD features in NK cell-sufficient/deficient PBS-OVA pups were approximately of the same level as AAD features in NK cell-deficient DEP-OVA pups. Together, our results indicated that NK cell engagement in AAD was not merely a consequence of allergen exposure or young age of pups, but a consequence of maternal exposure to DEP.

The NK cell granule protease granzyme B induces IL-25 in airway epithelial cells. IL-13 is a recognized inducer of epithelial IL-25 (23), providing one potential link between NK cells and IL-25. However, IL-13 can be produced by other cells, suggesting that the requirement for NK cells in AAD is imposed by another mediator or mediators more specific to NK cells. In search of these mediators, we analyzed previously published NK cell transcriptome data $(15,18$, 24); selected mRNAs with links to airway epithelial cells, the lung, asthma, or type 2 immunity; and measured them in lungs of DEPOVA Nori ${ }^{\mathrm{C} r e /+} R 26^{\mathrm{DTA} /+}$ and $R 26^{\mathrm{DTA} /+}$ littermate pups (Figure $8 \mathrm{~A}$ ). None of these mRNAs were affected by NK deficiency, suggesting greater importance of other cellular sources. In contrast, NK cell-related mediators of cytotoxicity, i.e., the proteases granzyme A, granzyme B, and cathepsin $\mathrm{W}$, were markedly reduced. A large reduction of granzymes indicated that NK cells were their primary source. Indeed, NK cells accounted for $88.28 \% \pm 0.99 \%$ of granzyme $\mathrm{B}^{+}$cells in lungs of DEP-OVA pups (Figure $8 \mathrm{~B}$ ). This result was consistent with previous reports comparing granzyme levels 

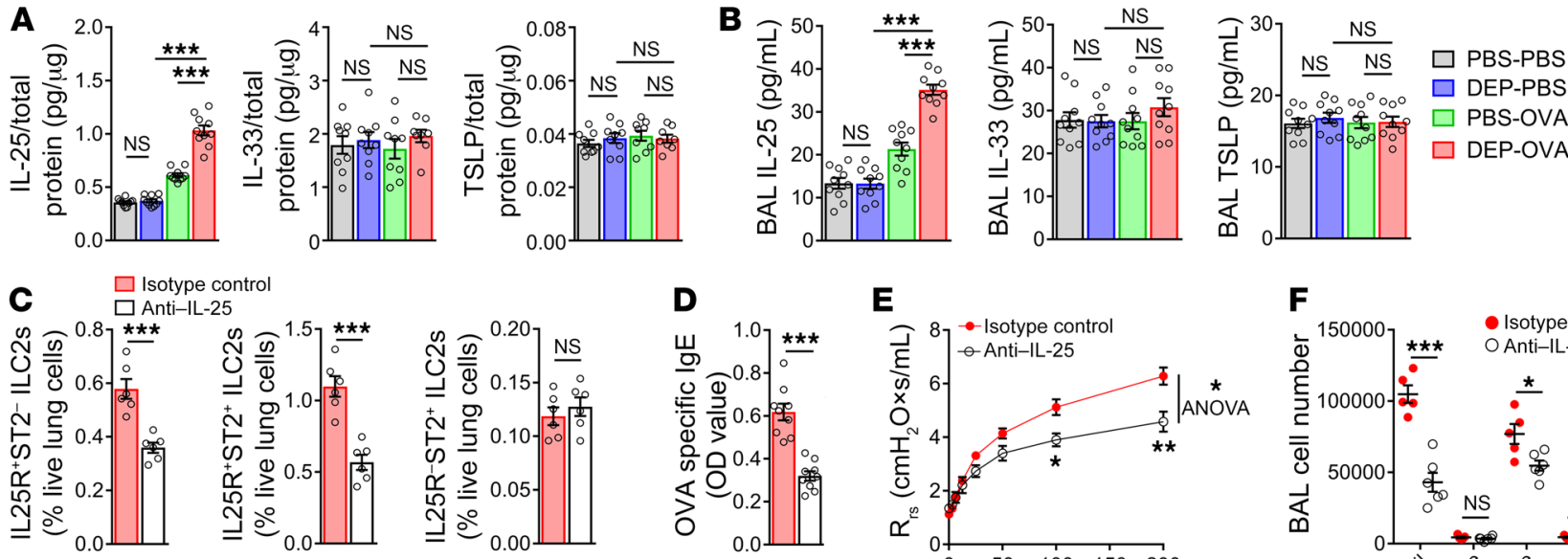

E
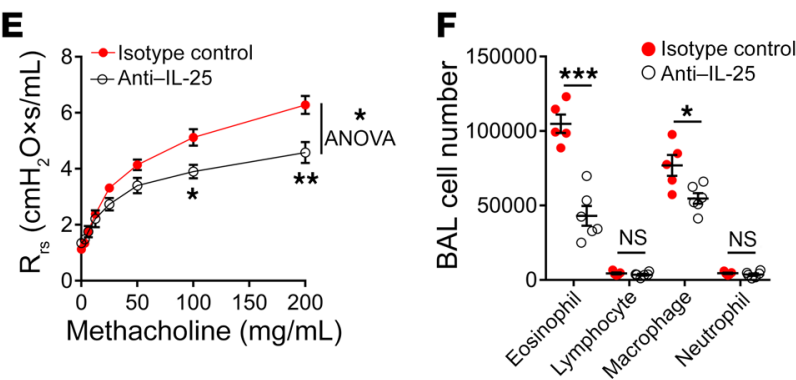

G

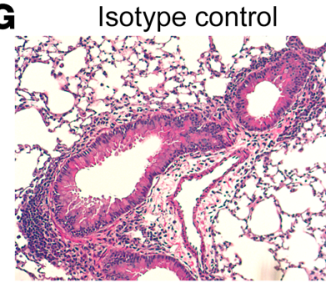

Anti-IL-25
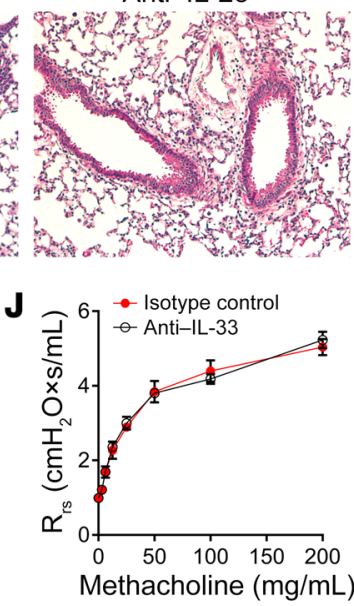

M
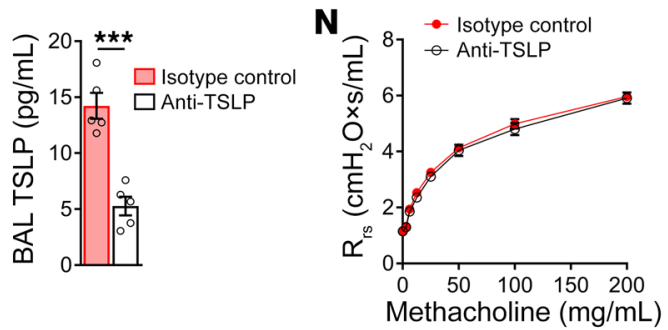

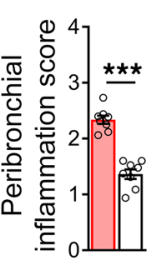

H
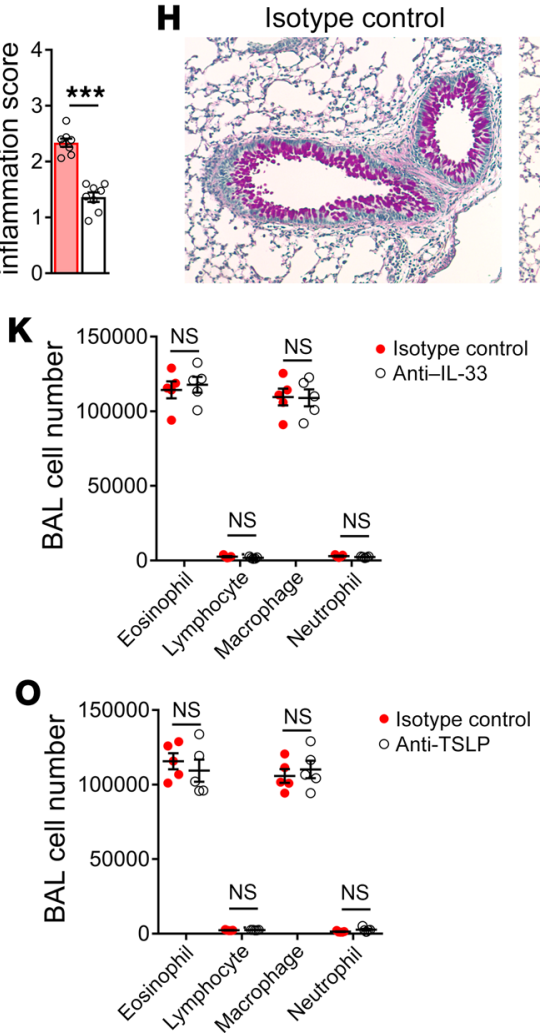

Anti-IL-25
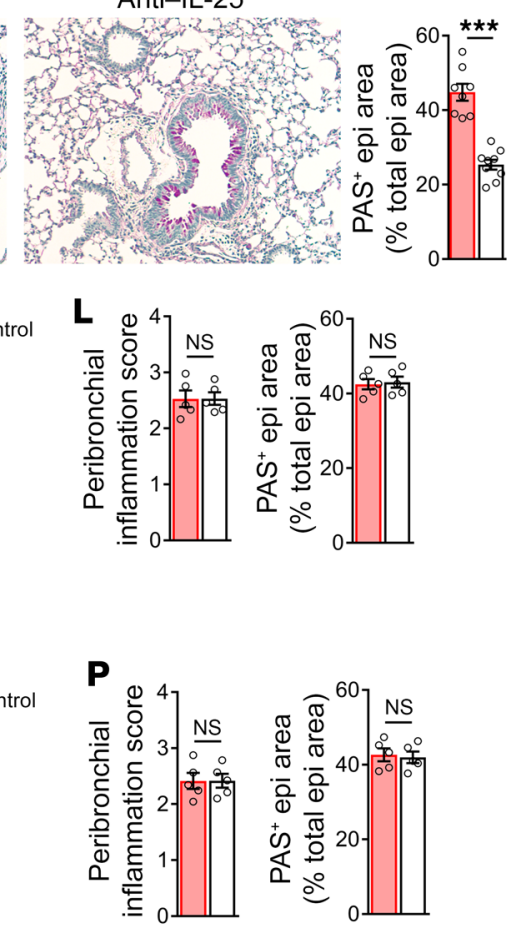

Figure 4. The type 2 immune response and AAD are dependent on IL-25. (A and $\mathbf{B})$ Concentrations of IL-25, IL-33, and TSLP in lung homogenates (A, $n=$ 10 [IL-25] or $n=9$ [IL-33, TSLP]) and BAL fluid (B, $n=10)$ from PBS-PBS, DEP-PBS, PBS-OVA, and DEP-OVA pups. (C-P) Depletion of IL-25 (C-H), IL-33 (I-L), and TSLP (M-P) in DEP-OVA pups. Pups received an anti-cytokine (anti-IL-25/anti-IL-33/anti-TSLP) antibody or isotype control IgG before immunization and then before the first challenge with OVA and were analyzed 72 hours after the final challenge (diagram of experimental strategy in Supplemental Figure 4B). (C) Percentages of IL25R+ST2- ILC2s, IL25R+ST2+ ILC2s, and IL25R-ST2+ ILC2s in live lung cells. $n=6$. (D) OVA-specific IgE in the serum. $n=9$. (E, J, and $\mathbf{N}$ ) Total lung resistance to methacholine (FlexiVent). $n=5-6$. (F, $\mathbf{K}$, and $\mathbf{O}$ ) Leukocyte subset counts in BAL fluid. $n=5-6$. (G, H, L, and $\mathbf{P})$ Images of H\&E-stained lung sections. Original magnification, $\times 100$. (G) Peribronchial inflammation scores $(\mathbf{G}, \mathbf{L}$, and $\mathbf{P})$, images of PAS-stained lung sections (H), and proportions of bronchial epithelial areas that are PAS (mucin)+ $(\mathbf{H}, \mathbf{L}$, and $\mathbf{P}$ ). $n=8-9$ (anti-IL-25); $n=5$ (anti-IL-33 and anti-TSLP). (I and $\mathbf{M}$ ) Concentrations of IL-33 (I) and TSLP (M) in BAL fluid. $n=5$. Data are representative of $2(\mathbf{A}, \mathbf{B}, \mathbf{E}$, and $\mathbf{I}-\mathbf{P})$ or $\mathbf{3}(\mathbf{C}, \mathbf{D}$, and $\mathbf{F}-\mathbf{H})$ independent experiments and are shown as mean \pm SEM. ${ }^{*} P<0.05 ;{ }^{* *} P<0.01 ;{ }^{* *} P<0.001$, 1-way ANOVA with Tukey's post hoc test (A and $\left.\mathbf{B}\right) ; 2$-tailed unpaired $t$ test (C, D, F-I, K-M, $\left.\mathbf{O}, \mathbf{P}\right)$; and 2-way repeated-measures ANOVA with Bonferroni's post hoc test (E, J, and $\mathbf{N})$.

in NK cells versus $\mathrm{CD}^{+} \mathrm{T}$ cells. NK cells have much higher expression of granzymes at baseline as part of their persistently alert state (18). $\mathrm{CD}^{+} \mathrm{T}$ cells require stimulation to induce granzymes. This stimulation was perhaps insufficient in our model.
We tested to determine whether granzymes and cathepsin $\mathrm{W}$ could directly stimulate airway epithelial cells to produce IL-25. To this end, we incubated C57BL/6 mouse primary airway epithelial cells (for purity; see Supplemental Figure 8A) with or with- 
A $\square R 26^{D T A++}$
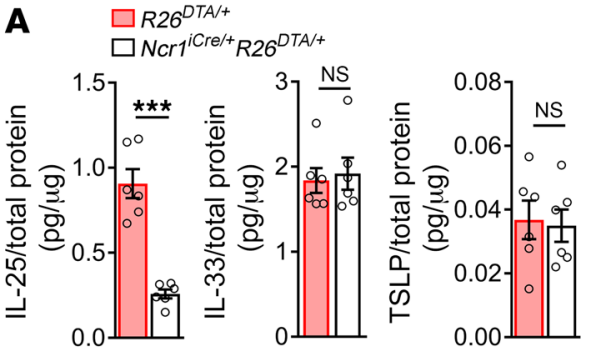

B
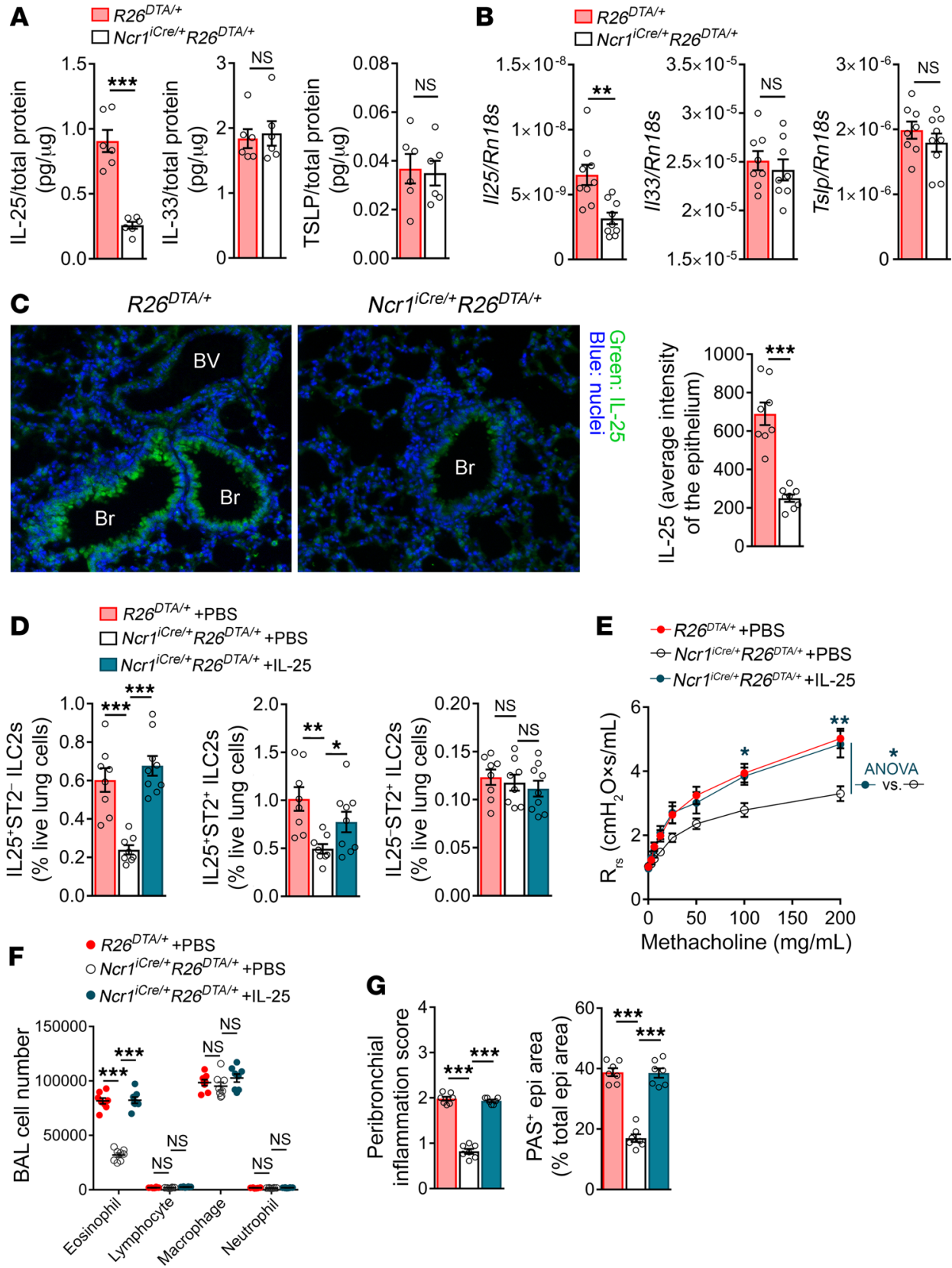

E

$$
\begin{aligned}
& -R 26^{\mathrm{DTA}+}+\mathrm{PBS} \\
& -\mathrm{Ncr} 1^{\mathrm{ICr} / \mathrm{H}} \mathrm{R} 26^{\mathrm{DTA+}+}+\mathrm{PBS}
\end{aligned}
$$$$
\text { - Ncricrel+t } R 26^{\text {DTA }+}+\mathrm{IL}-25
$$

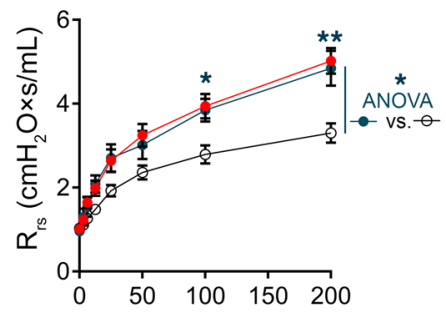

Methacholine $(\mathrm{mg} / \mathrm{mL})$
G

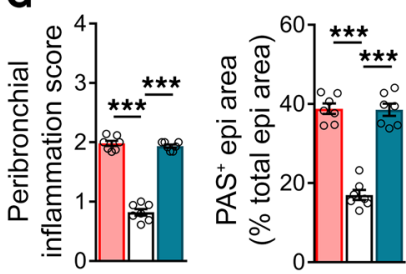

Figure 5. NK cells are upstream of IL-25. (A-C) IL-25, IL-33, and TSLP in DEP-OVA Ncri ${ }^{\text {ire/+ }} R 26^{\text {DTA } /+}$ and $R 26^{\text {DTA/+ }}$ littermates. (A) Concentrations of cytokines in lung homogenates. $n=6$ mice per group. (B) Levels of cytokine mRNAs in lung lysates. Rn18s, $18 \mathrm{~S}$ ribosomal RNA. $n=8-9$. (C) Left: fluorescent microscopy images of lung sections stained with an anti-IL-25 antibody (green) and the nuclear dye DAPI (blue). Br, bronchus; $B V$, blood vessel. Original magnification, $\times 200$. Right: average fluorescence intensity of the IL-25 signal in the airway epithelium. $n=8$. (D-G) IL-25 reconstitution. Pups were produced as in Figure 3, K-N. On postnatal day 5, pups were injected with OVA/alum mixed with IL-25 or PBS. IL-25/PBS injections were repeated on day 6 , but this time no OVA/alum was used. All pups were OVA challenged on days 23 to 25 . Six hours after each challenge, pups were i.t. administered IL-25 or PBS. Pups were analyzed on day 28 (diagram of experimental strategy in Supplemental Figure 5). (D) Percentages of IL25R+ST2- ILC2s, IL25R+ST2+ ILC2s, and IL25R-ST2+ ILC2s in live lung cells. $n=8-9$. (E) Total lung resistance to methacholine. $n=7$. (F) Leukocyte subset counts in BAL fluid. $n=8$. (G) Peribronchial inflammation scores and proportions of bronchial epithelial areas that are $\mathrm{PAS}^{+} . n=7$. Data are representative of 3 independent experiments and are shown as mean \pm SEM. ${ }^{*} P<0.05 ;{ }^{* *} P<0.01$; ${ }^{* * *} P<$ 0.001, 2-tailed unpaired $t$ test (A-C); 1-way ANOVA with Tukey's post hoc test (D, F, and G); and 2-way repeatedmeasures ANOVA with Bonferroni's post hoc test (E). out recombinant mouse granzyme A, granzyme B, or cathepsin $\mathrm{W} \pm \mathrm{IL}-13$, or with an extract of an allergen Alternaria alternata (positive control) (Figure 8, C and D). The A. alternata extract is a well-established activator of airway epithelial cells, inducing release of several proinflammatory mediators, including IL-25. Granzyme B induced IL-25 (Figure 8, C and D). Furthermore, granzyme B potentiated the effect of IL-13 on IL-25, highlighting cooperation between these 2 NK cell-expressed epithelial activators. Experiments using small molecule inhibitors indicated that granzyme B-mediated IL-25 induction was dependent on the protease-activated receptor 2 (PAR2), emphasizing importance of granzyme B proteolytic activity (Figure $8 \mathrm{E}$ ). The inhibitor experiment also pointed to substrate selectivity of the granzyme $B$ protease, the action of which was not dependent on another member of the PAR family - PAR1. Granzyme A and cathepsin
W did not have any effects on IL-25, consistent with previous reports showing that granzyme A, granzyme B, and cathepsin $\mathrm{W}$ differ in their substrate specificities (25). None of the proteases affected IL-33 and TSLP. In control experiments, we determined that all proteases were enzymatically active (Supplemental Figure $8 \mathrm{~B})$, indicating that inability of granzyme A and cathepsin $\mathrm{W}$ to induce IL-25 was not due to absence of their proteolytic activity. We also determined that none of the proteases induced cell death (Figure 8F), excluding a possibility of death-related release of IL-25. Lack of cell death was consistent with an established paradigm on requirement of perforin and intracellular delivery for granzyme-mediated cell death (death-related substrates of granzymes [caspases] are intracellular) (26). Our culture system did not include perforin. Altogether, we identified granzyme B as an inducer of IL-25 in airway epithelial cells. Granzyme B showed 
$\square$ PBS

$\square$ PBS NK cells

A $\square$ DEP NK cells

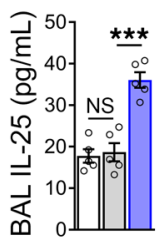

B

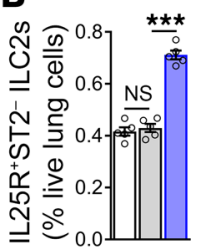

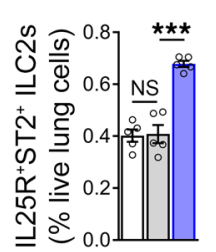
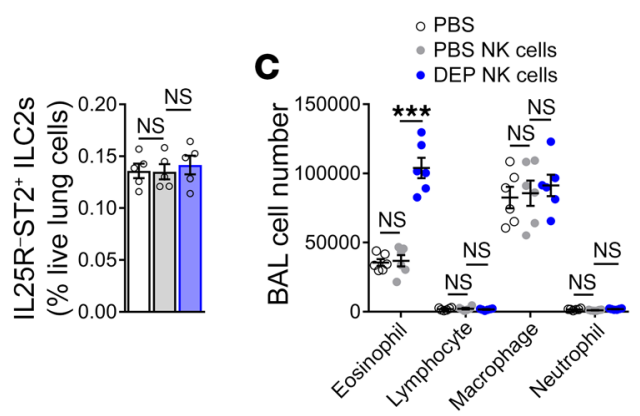

D

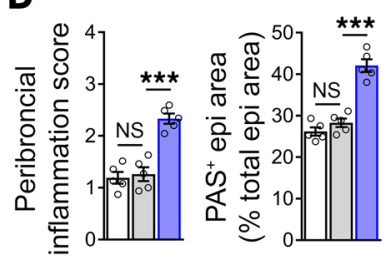

$\square$ PBS
$\square$ PBS NK cells
$\square$ DEP NK cells

E

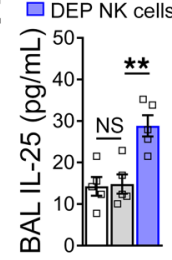

$\mathbf{F}$

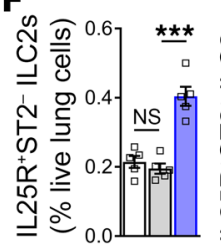

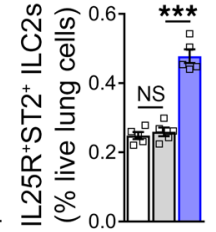
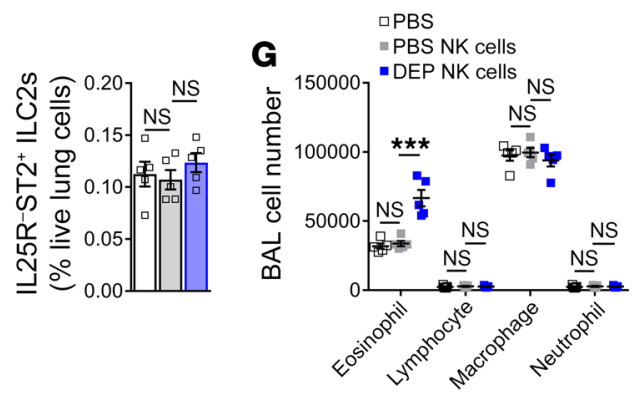

H

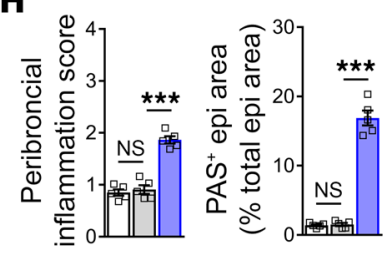

Figure 6. NK cells as carriers of susceptibility to AAD. (A-H) Transfer of NK cells from WT pups of DEP-exposed and PBS-exposed mothers (DEP NK cells and PBS NK cells, respectively) into $\mathrm{NCr}^{i \mathrm{ire} /+} R 26^{\mathrm{DTA} /+}$ pups of unexposed mothers. Prospective recipient pups were immunized with OVA/alum on postnatal day 5 (protocol 1, A-D) or postnatal day 26 (protocol 2, E-H). Donor pups were not immunized. Transfer of CD127- NK cells or PBS injection took place on day 22 (protocol 1) or day 43 (protocol 2), as in Figure 3, K-N. Recipients were challenged with OVA on days 23 to 25 (protocol 1) or days 44 to 46 (protocol 2) and analyzed on day 28 (protocol 1) or day 49 (protocol 2) (diagram of experimental strategy in Supplemental Figure 6). (A and E) Concentration of IL-25 in the BAL fluid of recipients. (B and F) Percentages of IL25R+ST2- ILC2s, IL25R+ST2+ ILC2s, and IL25R-ST2+ ILC2s in live lung cells of recipients. (C and $\mathbf{G}$ ) Leukocyte subset counts in BAL fluid of recipients. ( $\mathbf{D}$ and $\mathbf{H})$ Peribronchial inflammation scores and proportions of bronchial epithelial cell areas that are PAS $. n=6$ (protocol 1); $n=5$ (protocol 2). Data are representative of 2 independent experiments and are shown as mean $\pm S E M$. ${ }^{* *} P<0.01 ;{ }^{* * *} P<0.001$, 1-way ANOVA with Tukey's post hoc test.

relative selectivity toward IL-25, as IL-33 and TSLP were not affected by its action. Our data confirmed previous observations on differences in regulation of these cytokines (27-32). To exemplify this, IL-33 is constitutively expressed at a high level by various epithelial cells from multiple organs, endothelial cells of high endothelial venules (HEV), and fibroblastic reticular cells (FRC) of lymph nodes and spleen (27). Within pulmonary epithelium, IL-33 is primarily expressed by human basal cells and mouse alveolar type 2 cells. Constitutive expression of IL- 25 is limited to tuft cells, a rare epithelial subset that is seen in the gut, upper airways, and trachea, but not in the distal airways and the lung (28-30); other cells, including bronchial epithelial cells, require environmental stimulation to produce IL-25. The dominant mechanism by which IL-33 is released from cells is necrosis. Only few stimuli are known to liberate IL-33 from living cells, and these include the A. alternata extract (working via coordinated activation of multiple receptors and pathways), ATP, and mechanical stress $(31,32)$. In contrast, IL-25 secretion can be elicited by less complex stimuli, such as cytokines (ref. 23 and Figure 8D).

NK cells promote IL-25 production through direct effects on the airway epithelium. Our experiments indicated that at least $2 \mathrm{NK}$ cell mediators (the granule protease granzyme B and IL-13) had the capacity to directly act on the airway epithelium and induce IL-25. We therefore wondered whether induction of IL- 25 by NK cells occurred through their direct effects on epithelial cells. To answer this question, we set up an in vitro coculture system using normal C57BL/6 airway epithelial cells (as in Figure 8, C-F) and NK cells that were isolated from spleens of DEP-OVA pups. DEP-OVA NK cells induced the IL-25 protein in epithelial cells (Figure $8 \mathrm{G}$ ).

Granzyme $B$ is required for development of $A A D$ in predisposed pups. We next asked whether granzyme B was important for development of AAD in our model. To this end, we performed 2 experiments. In the first experiment, we studied the role of systemic granzyme B. DEP-exposed females heterozygous for knockout mutation in the granzyme B gene $\left(\mathrm{Gzmb}^{-/+}\right)$were mated with unexposed $\mathrm{Gzmb}^{-/+}$males to produce $\mathrm{Gzmb}^{-/-}$and $\mathrm{Gzmb}^{+/+}$littermate pups. Pups were immunized and challenged with OVA, per our regular protocol. Compared with $\mathrm{Gzmb}^{+/+}$pups, $\mathrm{Gzmb}^{-/-}$pups had less IL-25 and fewer IL25R $\mathrm{R}^{+}$ILC2s in their lungs (Figure 9, A and B) and reduced AAD (Figure 9, C-F). In the second experiment, we explored the role of NK cell-expressed granzyme B (diagram of the experimental strategy in Supplemental Figure 9). This was accomplished through transfer of $\mathrm{Gzmb}^{-/-}$and control $\mathrm{Gzmb}^{+/+} \mathrm{CD} 127^{-} \mathrm{NK}$ cells into NK cell-deficient recipients. Donor $\mathrm{Gzmb}^{-/-}$and $\mathrm{Gzmb}^{+/+}$ pups were littermates born to $\mathrm{Gzmb}^{-/+}$parents. Recipient pups came from the cross of $\mathrm{Ncr}^{\mathrm{iCr} /+}$ mothers and $\mathrm{R} 26^{\mathrm{DTA} / \mathrm{DTA}}$ fathers. Both sets of mothers (generating either donors or recipients) received DEP. Prospective donor and recipient pups were immunized with OVA in alum on postnatal day 5. NK cell transfer (i.t. route) took place on postnatal day 22. After transfer, recipients were challenged with OVA. Compared with recipients of $\mathrm{Gzmb}^{+/+} \mathrm{NK}$ cells, recipients of $\mathrm{Gzmb}^{-/-}$NK cells had less IL-25 and fewer IL25R ILC2s (Figure 9, $\mathrm{G}$ and $\mathrm{H}$ ) and decreased AAD (Figure 9, I-L). In conclusion, NK cell-expressed granzyme $\mathrm{B}$ was required for elicitation of AAD in 
A $\square$ Adult R26 $6^{\text {TA+ }+}$

$\square$ Adult $\mathrm{Ner}{ }^{\text {iCrelt }} \mathrm{R} 26^{\mathrm{DTA}+}$

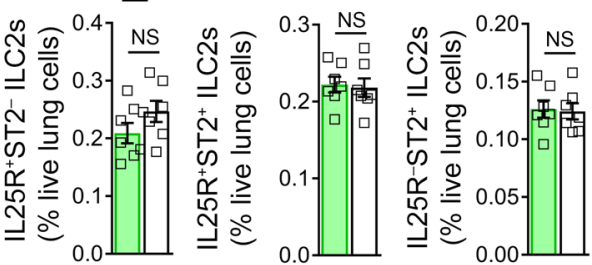

B

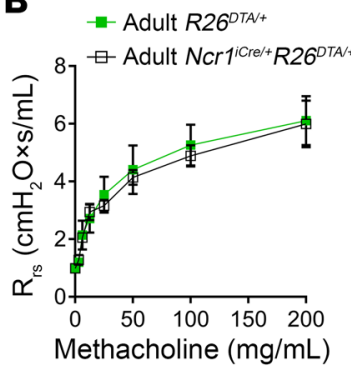

C $\quad$ Adult $R 26^{\text {DTA }+}$

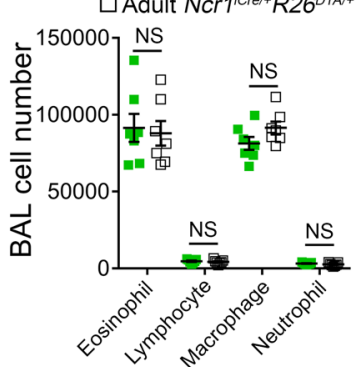

D

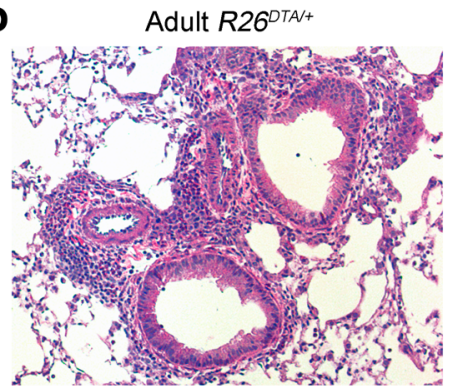

Adult $\mathrm{Ncr} 1^{\mathrm{iCre} /+} \mathrm{R} 26^{\mathrm{DTA}+}$
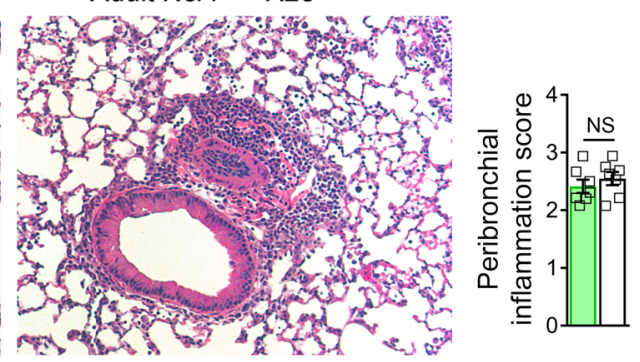

E

Adult R26

Adult $\mathrm{Ncr} 1^{\mathrm{iCre} /+} \mathrm{R} 26^{\mathrm{DTA} /+}$
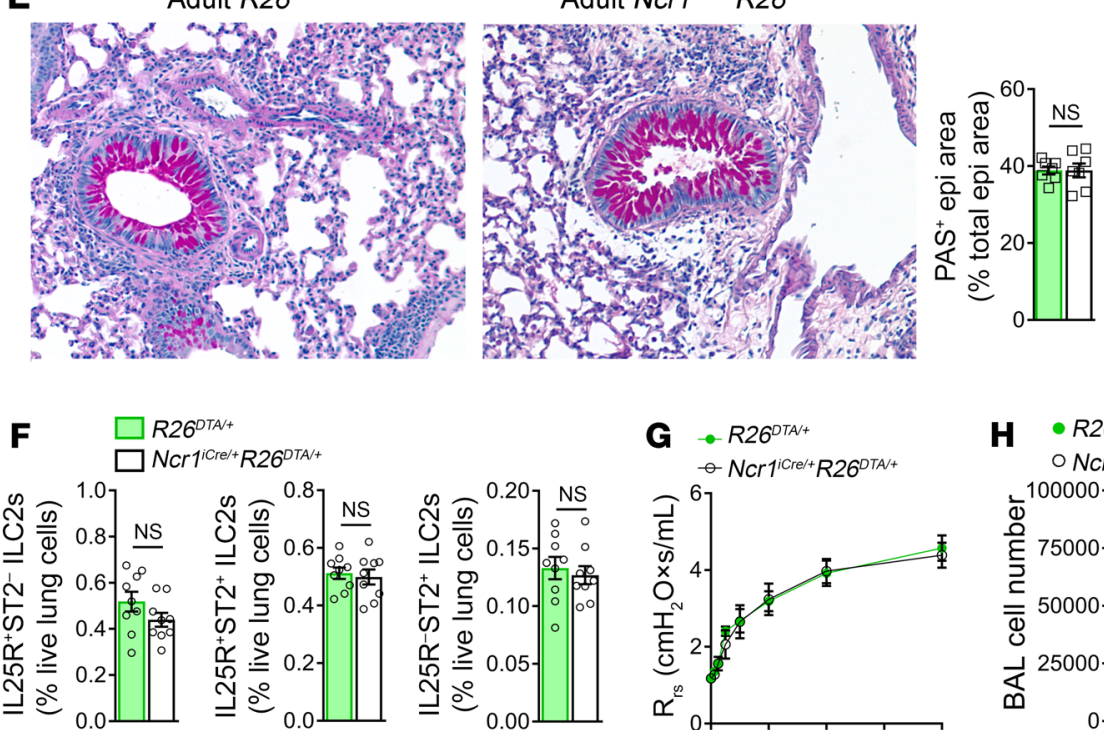

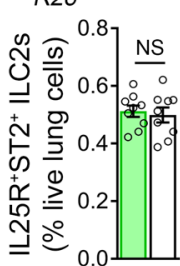

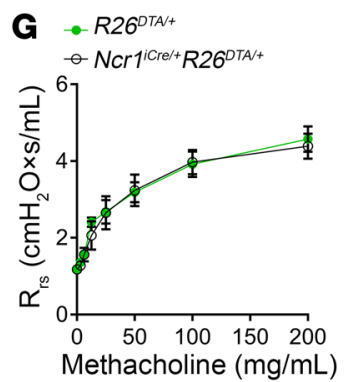

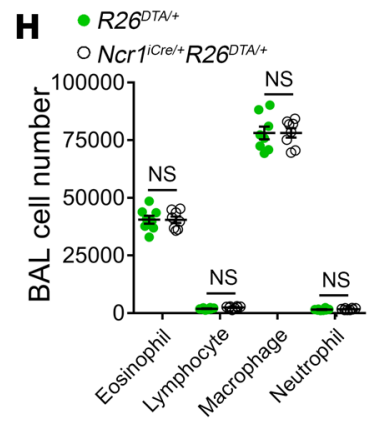

I
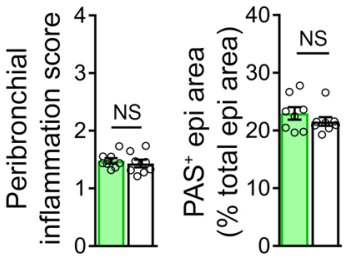

Figure 7. NK cell engagement is unique to maternal DEP-programmed AAD. (A-E) Adult mouse model of AAD. Unexposed Ncr1 ${ }^{i c r e /+}$ females were mated with unexposed $R 26^{D T A / D T A}$ males. Ncr ${ }^{i c r e /+} R 26^{D T A /+}$ and $R 26^{D T A /+}$ offspring were i.p. immunized with OVA in alum on postnatal days 42 and 49 , i.n. challenged with OVA on days 56, 57, and 58, and analyzed on day 61 (diagram of experimental strategy in Supplemental Figure 7). (F-I) Neonatal mouse model of AAD. PBS-exposed Ncricre/+ females were mated with unexposed $R 26^{\text {DTA/DTA }}$ males to produce Ncr ${ }^{i \mathrm{Cr} /+} R 26^{\mathrm{DTA} /+}$ and $R 26^{\mathrm{DTA} /+}$ littermates. Pups were immunized with OVA/alum on postnatal day 5, challenged with OVA on days 23 to 25 , and analyzed on day 28 . (A and F) Percentages of IL25R ${ }^{+}$ST2- ILC2s, IL25R+ST2+ ILC2s, and IL25R-ST2+ ILC2s in live lung cells. $n=7$ mice per group (A); $n=9$ (F). (B and $\mathbf{G})$ Total lung resistance to methacholine. $n=6(\mathbf{B}) ; n=8$ (C). (C and $\mathbf{H})$ Leukocyte subset counts in BAL fluid. $n=7$ (C); $n=8$ (H). (D) Left: H\&E-stained lung sections. Original magnification, $\times 100$. Right: peribronchial inflammation scores. $n=7$. (E) Left: PAS-stained lung sections. Original magnification, $\times 100$. Right: proportions of bronchial epithelial cell areas that are PAS $. n=7$. (I) Peribronchial inflammation scores and proportions of bronchial epithelial areas that are PAS $. n=8$. Data are representative of 3 (A-I) independent experiments and are shown as mean \pm SEM. Two-tailed unpaired $t$ test (A, B, D-F, and $\mathbf{H}-\mathbf{I}) ; 2$-way repeated-measures ANOVA with Bonferroni's post hoc test (C and $\mathbf{G})$.

predisposed pups. Absence of granzyme B specifically in NK cells yielded effects on AAD similar to those of the absence of systemic granzyme B. This was consistent with NK cells being the dominant source of this protease in our model (Figure 8B).
Granzyme B is required for the direct effect of NK cells on epithelial cells. We next asked whether granzyme B mediated the direct effect of NK cells on airway epithelial cells. To address this, we again employed our NK-epithelial coculture system, but this time 
$\mathbf{A} \square R 26^{D T A /+} \square N K p 46^{i C r e / t} R 26^{D T A /+}$

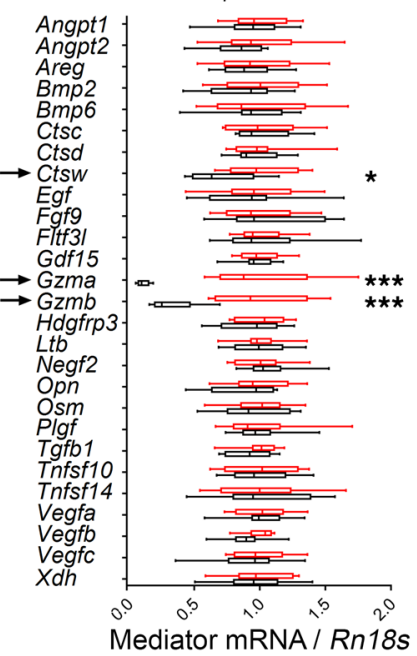

B

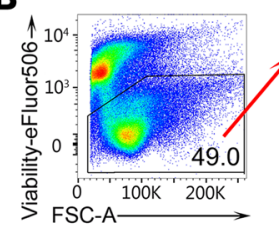

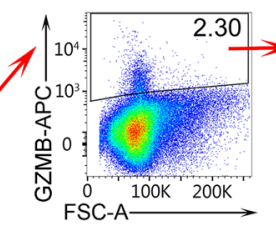
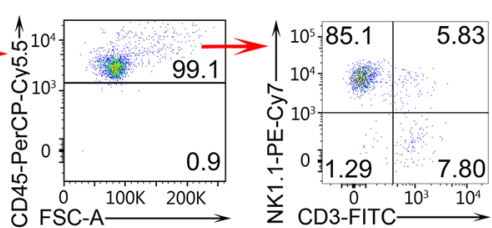

$\square$ CD45+CD3-NK1.1$\square \mathrm{CD}^{\circ} 5^{+} \mathrm{CD}^{+}{ }^{+} \mathrm{NK} 1.1^{+}$ $\square \mathrm{CD}^{\circ} 5^{+} \mathrm{CD}^{+} \mathrm{NK} 1.1^{-}$ $\square \mathrm{CD}^{\circ} 5^{+} \mathrm{CD}^{-} \mathrm{NK} 1.1^{+}$ $\square$ CD45-
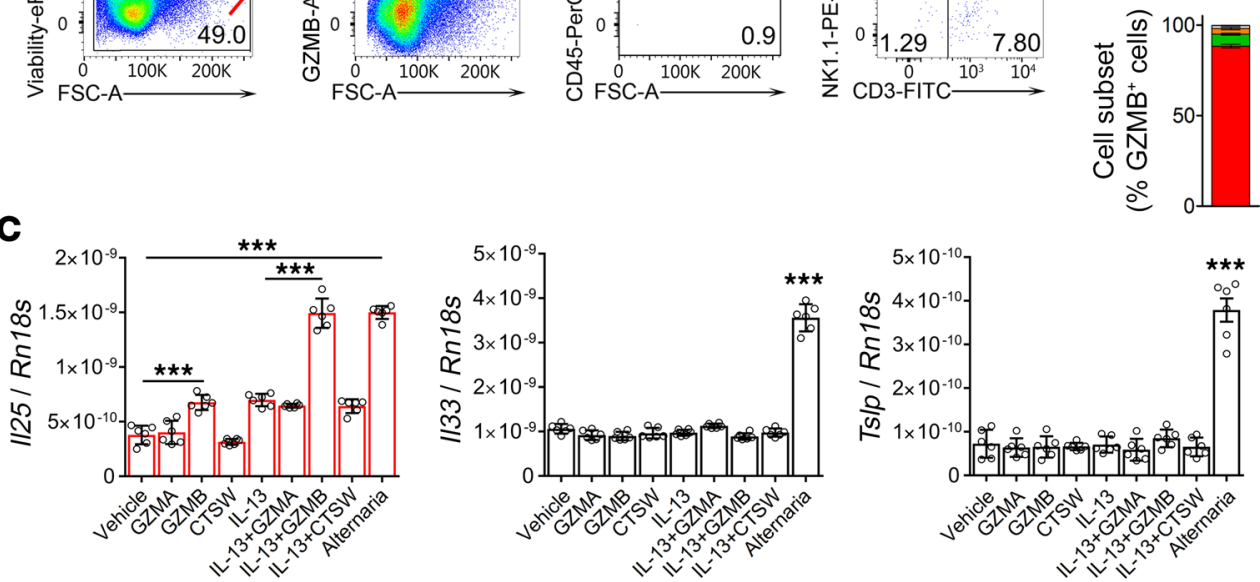
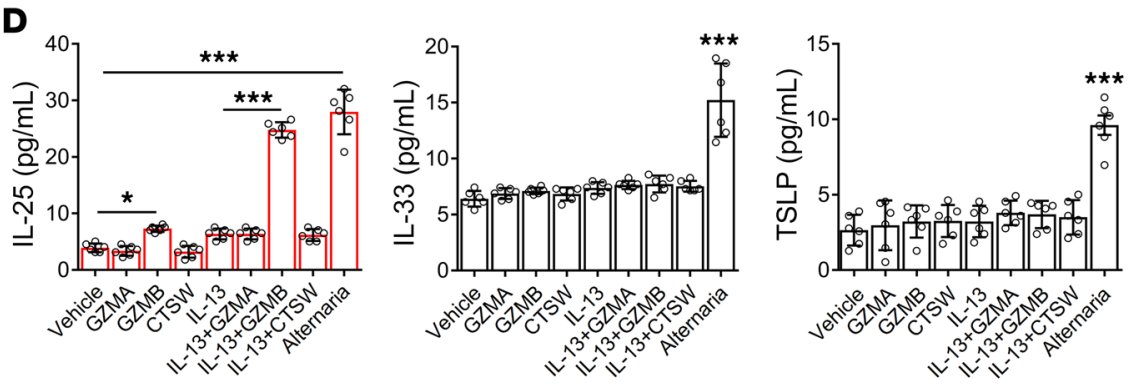

E
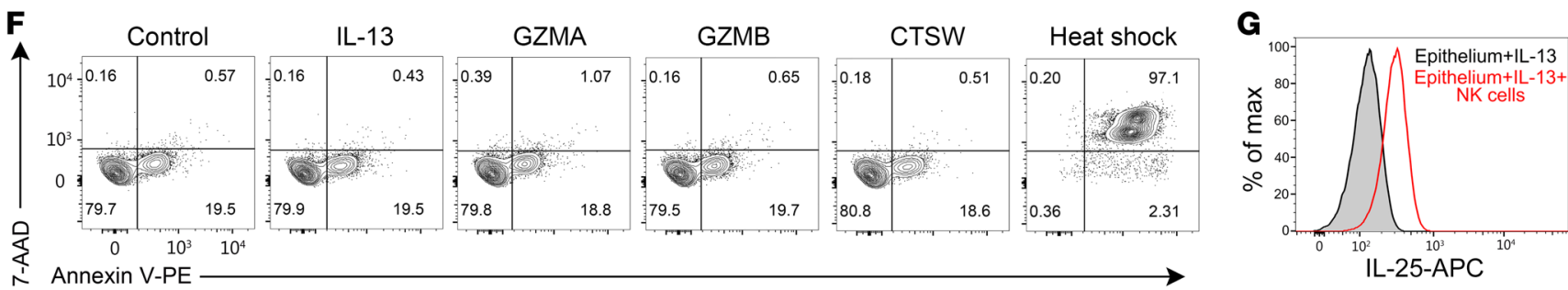

Figure 8. The NK cell granule protease granzyme B induces IL-25 in airway epithelial cells. (A) Levels of mediator mRNAs in lungs of DEP-OVA Ncricre/+ $R 26^{D T A /+}$ and $R 26^{D T A /+}$ littermates. $n=10$ mice per group. Lines within boxes represent medians, boxes represent 25 th to 75 th percentiles, and whiskers represent minimum and maximum values. (B) Left: gating strategy to define lung cell subsets that express granzyme B (CZMB). Right: proportions of indicated cell subsets in population of GZMB+ cells. $n=7$. (C and $\mathbf{D}$ ) Production of $/ 125,1 / 33$, and TsIp mRNAs (C; cell lysates; $n=6$ samples per group) and IL-25, IL-33, and TSLP proteins ( $\mathbf{D}$; culture supernatants; $n=6$ ) by C57BL/6 Mouse Primary Tracheal and Bronchial Epithelial Cells (Cell Biologics Inc.) treated with vehicle (PBS), granzyme A (CZMA), granzyme B, cathepsin W (CTSW) \pm IL-13, or extract of A. alternata. (E) II25 mRNA in epithelial cells treated with GZMB, GZMB + PAR2 inhibitor (CB83 or ENMD-1068), and GZMB + PAR1 inhibitor (SCH79797 or vorapaxar); vehicle 1 = PBS (vehicle for GZMB), vehicle 2 = DMSO (vehicle for inhibitors). $n=8$ (vehicle 1 + vehicle 2); $n=6$ (other conditions). (F) Flow cytometric detection of dead cells in cultures of epithelial cells treated as in $\mathbf{C}$ and $\mathbf{D}$ or subjected to heat shock (positive control for cell death). (C) Flow cytometric detection of IL-25 in EpCAM+NK1.1 epithelial cells incubated with IL-13 \pm NK cells from spleens of WT DEP-OVA pups. Histogram is representative of 3 independent cocultures. Data are representative of 2 (A and B) or 3 (C-C) independent experiments and are shown as mean \pm SEM. ${ }^{*} P<0.05 ;{ }^{* * *} P<0.001$, 2-tailed unpaired $t$ test (A); 1-way ANOVA with Tukey's post hoc test (C-E).

used $\mathrm{Gzmb}^{-/-}$and $\mathrm{Gzmb^{+/+ }}$ NK cells. NK cells were isolated from spleens of DEP-OVA Gzmb $b^{-/-}$and $G z m b^{+/+}$littermates. Compared with $G z m b^{+/+}$NK cells, Gzmb $b^{-/-}$NK cells had reduced capacity to induce IL-25 (Figure 9M) in airway epithelial cells, highlighting the importance of the protease in mediating direct effects of NK cells.

DEP-programmed NK cells drive the type 2 immune response and $A A D$ to the clinically relevant house dust mite allergen. In our final experiments, we tested to determine whether the NK cell-depen- dent pathway was operational and important in the human-relevant context. To address this, we used a clinically relevant allergen to elicit AAD in mice and employed human cell-culture systems. In the first experiment, we tested to determine whether offspring responses to the human allergen were affected by maternal exposure to DEP. To this end, pups of DEP-exposed or PBS-exposed mothers were sensitized with an i.n. application of the HDM on postnatal days 5 and 6 , followed by 3 i.n. challenges with HDM on 
A
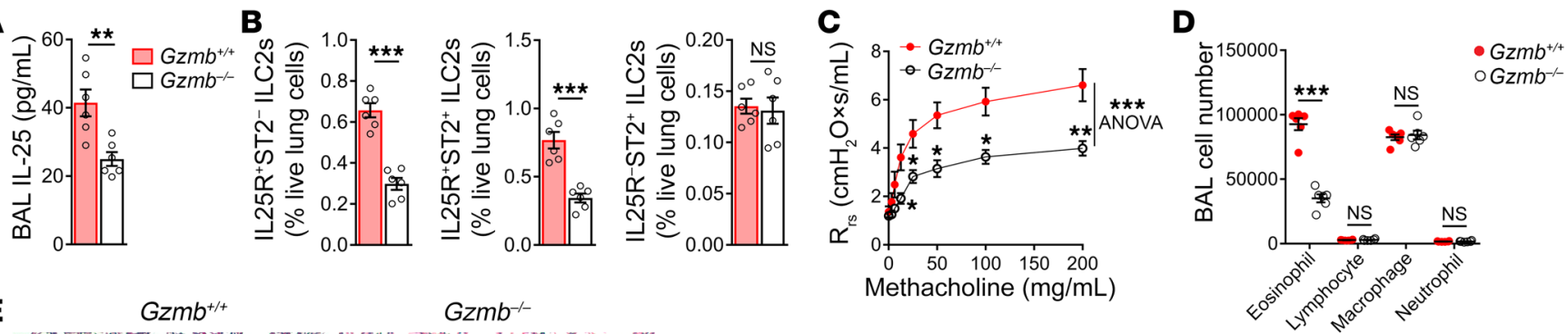

E

$\mathrm{Gzmb}^{+/+}$

$\mathrm{Gzmb}^{-1}$
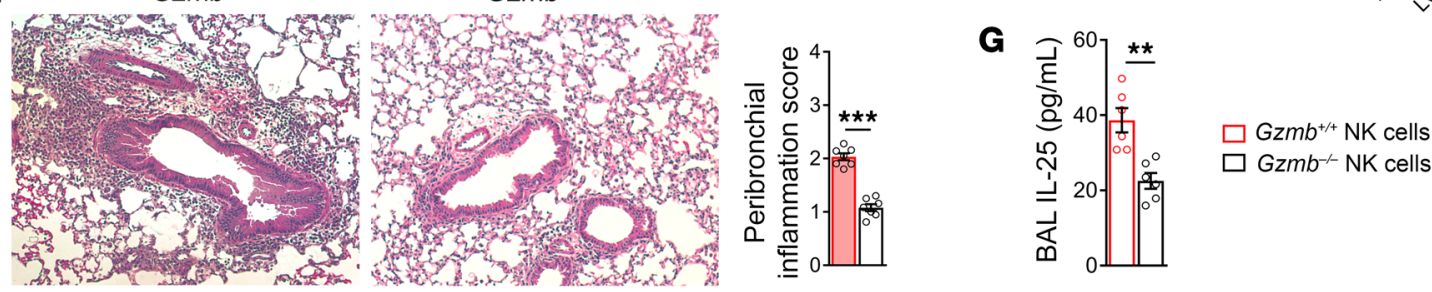

$\mathbf{F}$

$\mathrm{Gzmb+/+}$

$\mathrm{Gzmb}^{-1}$
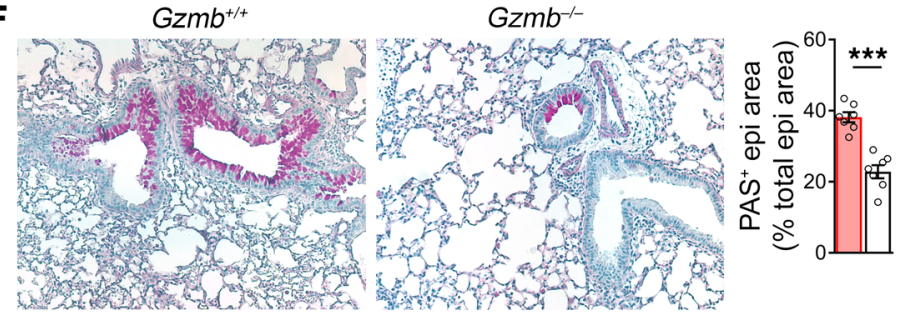

H

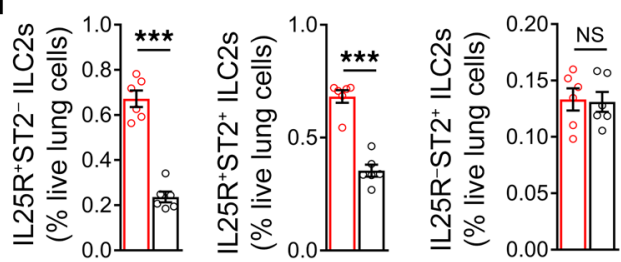

I

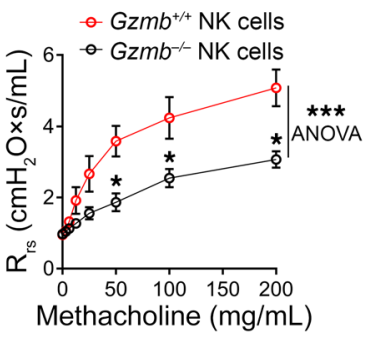

J
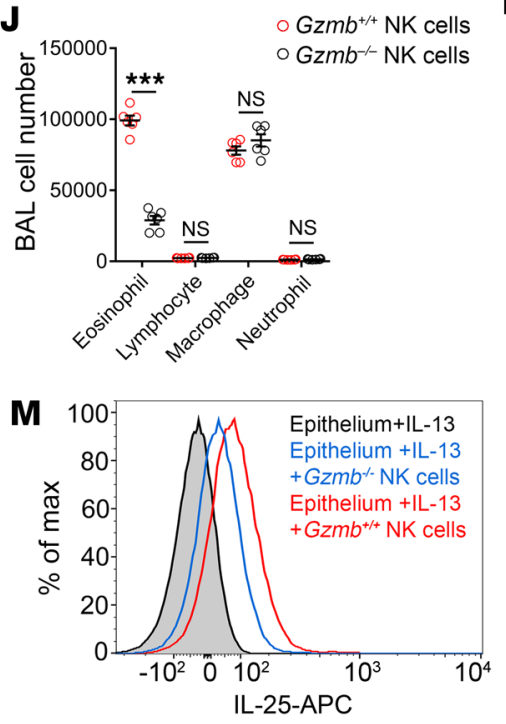

K $\quad \mathrm{Gzmb}^{+/+} \mathrm{NK}$ cells
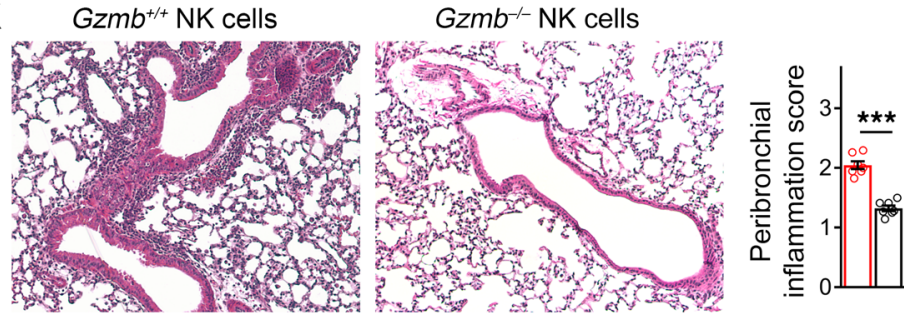

$\mathbf{L}$

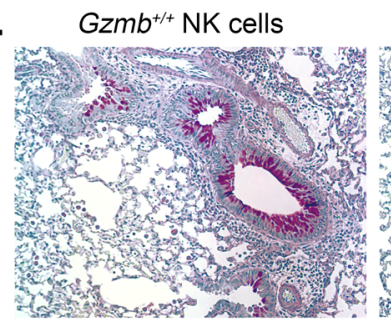

$\mathrm{Gzmb}^{-/-} \mathrm{NK}$ cells

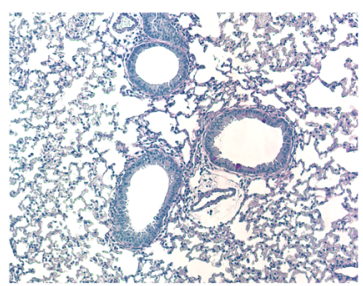

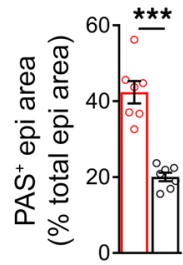

Figure 9. Granzyme $B$ is required for development of AAD in predisposed pups. (A-F) Importance of systemic granzyme B. DEP-exposed $\mathrm{Czmb}^{+/-}$females were mated with unexposed $\mathrm{Gzmb}^{+/-}$males to generate $\mathrm{Gzmb}^{-/-}$and $\mathrm{Gzmb} b^{+/+}$littermates. Pups were immunized, challenged with OVA, and analyzed 72 hours after challenge. (G-L) Importance of NK cell-expressed granzyme B. Ncr ${ }^{i \mathrm{Cr} /+}{ }^{2} 26^{\mathrm{DTA} /+}$ pups were transferred with $\mathrm{Gzmb}^{+/+}$and $\mathrm{Gzmb}^{-/-} \mathrm{NK}$ cells. Donor and recipients were the same age and were born to DEP-exposed mothers. Cell transfer took place on day 22 as in Figure 3, K-N. Before cell transfer, on postnatal day 5 , donor and prospective recipient pups were immunized with OVA/alum. After cell transfer, recipients were challenged with OVA on days 23 to 25 and analyzed on day 28 (diagram of experimental strategy in Supplemental Figure 9). (A and $\mathbf{G}$ ) Concentration of IL-25 in BAL fluid. $n=6$ mice per group. (B and $\mathbf{H}$ ) Percentages of IL25R+ST2- ILC2s, IL25R+ST2+ ILC2s, and IL25R-ST2+ ILC2s in live lung cells. $n=6$. (C and I) Total lung resistance to methacholine. $n=6$. ( $\mathbf{D}$ and $\mathbf{J}$ ) Leukocyte subset counts in BAL fluid. $n=6$. (E and $\mathbf{K}$ ) Left: $\mathrm{H} \& \mathrm{E}$-stained lung sections. Original magnification, $\times 100$. Right: peribronchial inflammation scores. $n=$ 7. ( $\mathbf{F}$ and $\mathbf{L}$ ) Left: PAS-stained lung sections. Original magnification, $\times 100$. Right: proportions of bronchial epithelial cell areas that are PAS+. $n=7$. (M) Flow cytometric detection of IL-25 in EpCAM+NK1.1- airway epithelial cells that were incubated with IL-13 \pm NK cells from spleens of DEP-OVA Czmb ${ }^{+/+}$and $\mathrm{Czmb}^{-/-}$ pups. Histogram is representative of 3 independent cocultures. Data are representative of 3 independent experiments and are shown as mean $\pm \mathrm{SEM} .{ }^{*} P<0.05 ;{ }^{*} P<0.01$; ${ }^{* *} P<0.001$, 2-tailed unpaired $t$ test (A, B, D-H, and J-L); 2-way repeated-measures ANOVA with Bonferroni's post hoc test (C and I). 
days 23, 24, and 25 and analysis on day 28 (diagram of experimental strategy in Supplemental Figure 10). PBS-HDM pups displayed weak type 2 immune response (Figure 10, B-E) and low-level AAD (Figure 10, $\mathrm{F}-\mathrm{H}$ ), as expected from normal unprimed (no maternal exposure) neonates. Type 2 immune response and AAD were considerably enhanced in DEP-HDM pups (Figure 10, B-H). This response was linked to increased expression of type 2 cytokines by pulmonary NK cells (Figure 10A). Therefore, we concluded that maternal exposure to DEP facilitated allergic response to HDM in offspring. In the second experiment, we asked whether HDM-elicited AAD was dependent on NK cells. Compared with $R 26^{\text {DTA/+ }}$ pups of DEP-exposed mothers, their NK cell-deficient $\mathrm{Ncr}^{1 \mathrm{Cr} /+}$ $R 26^{\text {DTA } /+}$ littermates had reduced type 2 immune response (Figure 10, I-L) and AAD (Figure 10, M-O), indicating that DEP-programmed NK cells are critical drivers of AAD in the HDM context.

DEP promotes degranulation and type 2 cytokine production by human cord blood NK cells. In the third experiment, we asked whether DEP could regulate functions of human early life NK cells. To test this, we measured NK cell activation in DEP-stimulated cultures of human cord blood mononuclear cells (CBMCs). Control cultures were stimulated with HDM or treated with a vehicle (PBS). DEP promoted NK cell degranulation (i.e., secretion of granzyme B; Figure 11, A, B, and E) and NK cell production of type 2 cytokines IL-4 and IL-5 (Figure 11, C, D, F, and G). IL-13 was undetectable in this system (Figure 11D). DEP had no effect on NK cell production of IFN- $\gamma$ (Figure 11H). HDM did not influence any of the NK cell activation parameters (Figure 11, A-H). These results suggested that DEP endowed human early life NK cells with the capacity to stimulate airway epithelial cells (via granule-derived granzyme B and IL-4, which shares its epithelial receptor with IL-13) and other cells involved in the type 2 response, such as CD $4^{+}$T cells, ILC2s, B cells, and eosinophils (via IL-4 and IL-5). HDM did not have these effects on NK cells, which was consistent with our mouse data (Figure 7) showing that NK cells did not participate in AAD elicited by an allergen alone, i.e., in the absence of DEP.

Human granzyme B stimulates human airway epithelial cells to produce IL-25. In the final experiment, we examined effects of human recombinant granzyme B on human primary airway epithelial cells in the in vitro culture model. Human granzyme B induced transcription of the IL 25 gene (Figure 11I). The granzyme B effect on IL 25 was enhanced by the IL4R $\alpha /$ IL13R $\alpha 1$ ligand - IL-13. Granzyme B had no effect on IL33. Collectively, these results reflected our data obtained using the mouse epithelial culture system (Figure 8, C and D).

\section{Discussion}

Asthma is a heterogeneous disease that encompasses multiple endotypes (distinct mechanistic variants). Emergence of a particular endotype is dictated by several factors, including genotype, sex, environmental exposures, and age/developmental period when these exposures occur. Using a mouse model, we described an NK cell-dependent endotype of AAD that arose in early life as a result of maternal exposure to DEP. We then showed that NK cells induced AAD via activation of airway epithelial cells. NK cellderived granzyme B directly stimulated the epithelium to produce IL-25. Granzyme B-mediated induction of IL- 25 was enhanced by IL-13 that was cosecreted by NK cells. NK cell-induced epithelial IL-25 activated ILC2s and Th2 cells, leading to eosinophilic inflammation of airways and AAD. Finally, experiments using human cord blood and human airway epithelial cells suggested that DEP might induce an identical pathway in humans.

In fact, there is some evidence in prior literature that the NK cell-linked, early onset endotype of asthma does indeed exist in humans. One recent study showed that inner-city children who developed allergic asthma by age 7 were distinguished from children who did not develop asthma by higher levels of NK cell-related transcripts in PBMCs at age 2, i.e., in a period before asthma manifestation (33). The list of the most highly upregulated genes included granzyme B. Since inner-city children are exposed to high levels of air pollution, including DEP, this study gave a hint on a potential link between DEP and NK cells in humans. Air pollution-triggered activation of NK cells may persist beyond early childhood, as indicated by another study that detected increased levels of NK cell-related transcripts (including transcript for granzyme B) in PBMCs of inner-city asthmatics at age 8-11 (34).

Previous reports also support connection between early life asthma and IL-25. Rhinovirus (RV) infection is an important trigger of asthma in young children. RV promotes asthma partly via activation of the airway epithelium. A recent study showed that induction of epithelial IL-25 by RV was age dependent, occurring only in neonates and not in adult mice (35). RV-triggered IL-25 drove neonatal AAD. Given our results and the established paradigm placing NK cells as key responders to viruses, we speculate that IL-25 induction in RV-infected neonates is driven by NK cells. Therefore, RV-induced neonatal asthma may be another asthma endotype that depends on NK cells.

In addition to predicting another endotype of asthma, our study has the potential to pave the way for development of diagnostic tests for early detection of asthma predisposition. We postulate that these tests should measure type 2 cytokine ${ }^{+} \mathrm{NK}$ cells, granzyme B, and IL-25. Currently, cord blood IgE is the only biomarker with a potential to predict future allergic disease (36). However, IgE may not capture an innate component of asthma predisposition, priming of ILC2s. NK cells act upstream of both innate (ILC2s) and adaptive (Th2s/IgE) arms of the type 2 immune response. We thus propose that measurements of NK cell-linked biomarkers could complement IgE tests, being particularly useful in cases in which IgE is negative. NK cell biomarkers are also potentially more sensitive in detecting early changes than ILC2 markers, because NK cells act upstream of ILC2s. Finally, because ILC 2 counts are extremely low in the blood, measurements of NK cell-linked markers are markedly more practical.

Our study has implications for understanding basic mechanisms of the immune response. NK cells are traditionally viewed as cytotoxic cells that kill dangerous nonself targets, such as virally infected and transformed cells. More recent data indicate that NK cells can affect normal cells, including cells of the immune system. By secreting IFN- $\gamma$ and TNF- $\alpha$, NK cells contribute to activation of dendritic cells, macrophages, and T cells and induction of the type 1 immune response (37). NK cells also take part in resolution of the type 1 response through killing of participating immune cells (37). Much less is known about NK cell effects on the type 2 response. There are reports on type 2 cytokine-producing NK cells in human asthma (38), but significance of this for asthma has never been addressed. NK cells can kill eosinophils in vitro, suggesting that 
A
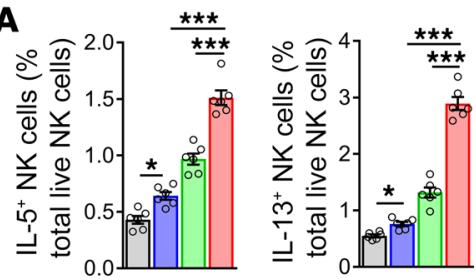

B
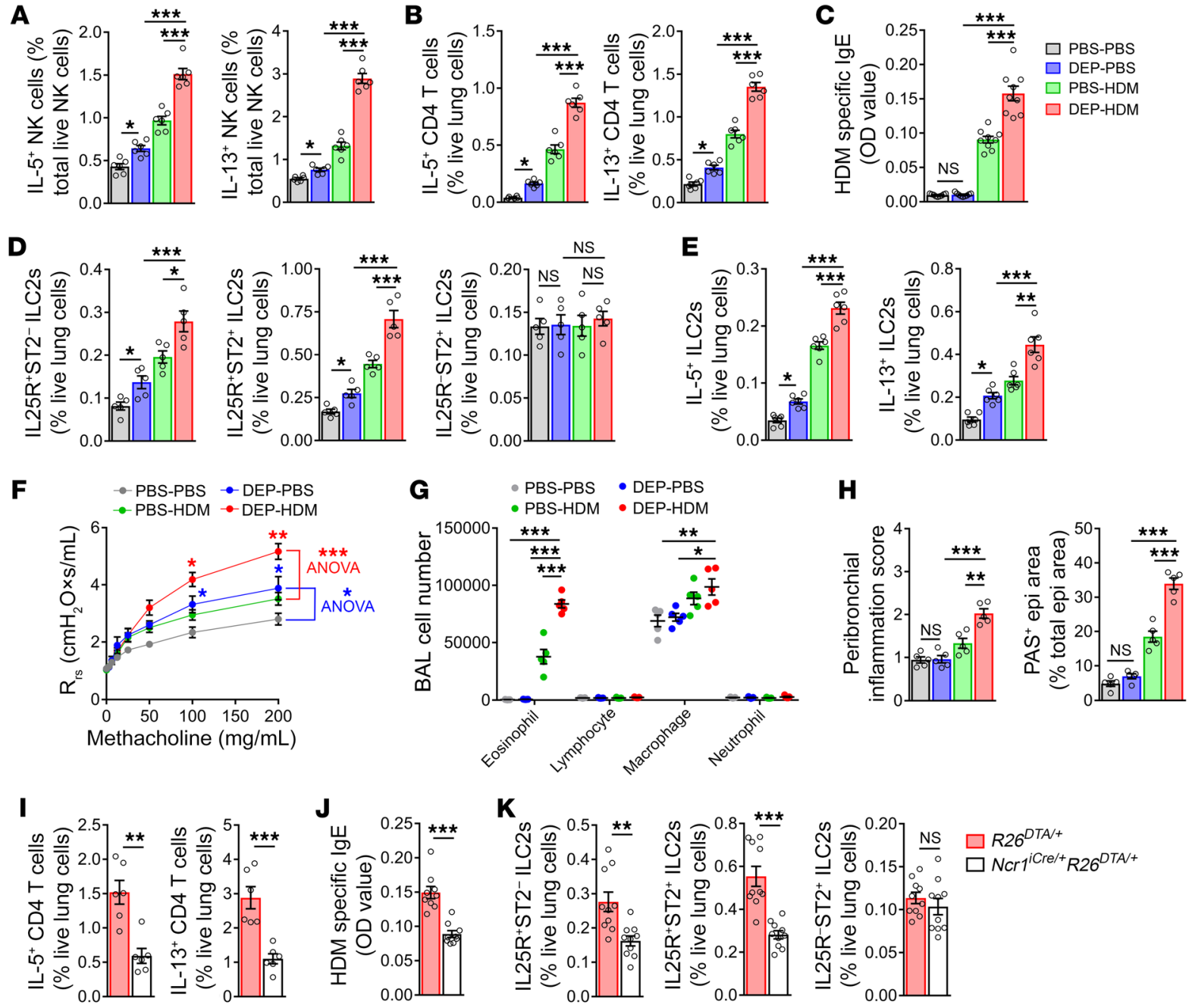

$\mathbf{K}$
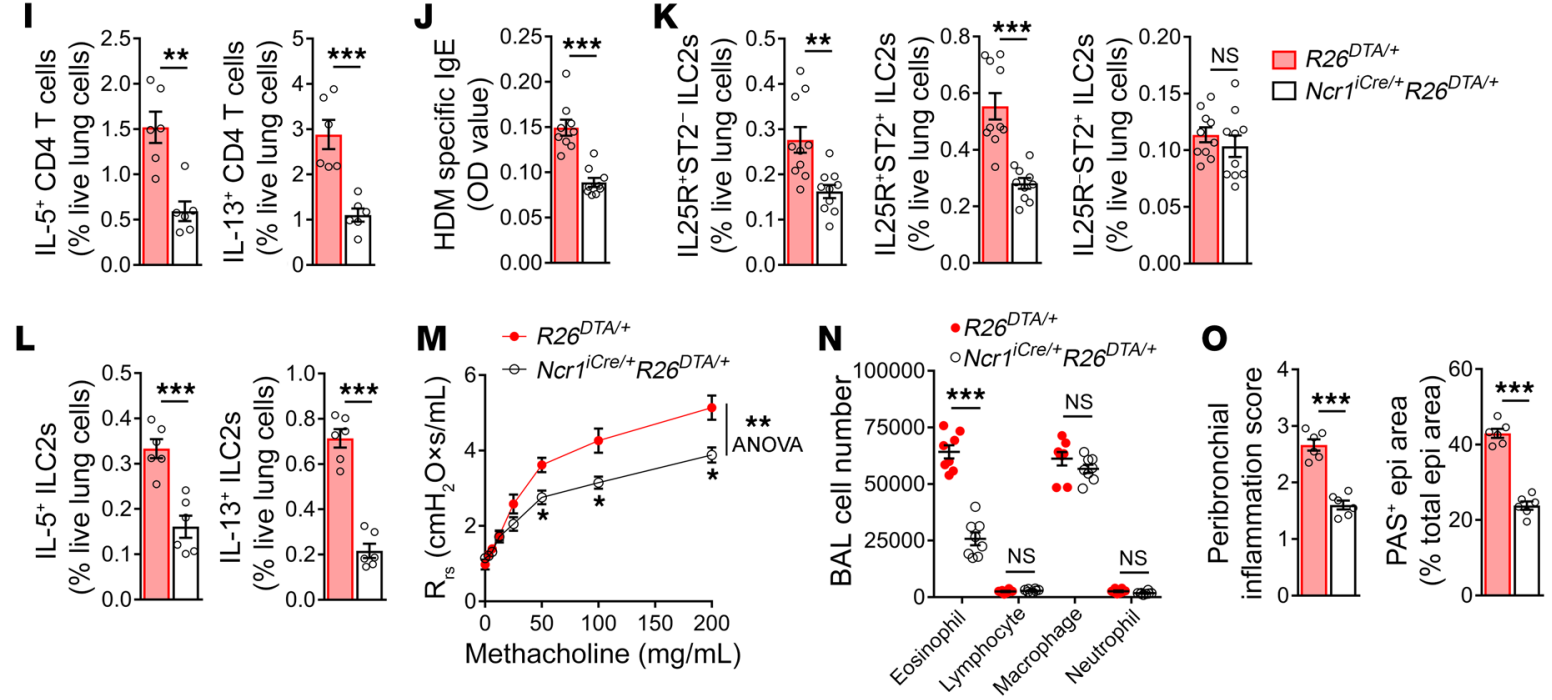

Figure 10. DEP NK cells drive HDM-induced AAD. (A-H) Model of early life HDM-induced AAD in the context of maternal exposure to DEP. Pups of DEP-exposed and PBS-exposed mothers received HDM intranasally on postnatal days 5 and 6 (immunization phase) and then on postnatal days 23 , 24, and 25 (challenge phase). Pups were analyzed on postnatal day 28 (diagram of experimental strategy in Supplemental Figure 10). (I-0) Genetic depletion of NK cells in DEP-HDM pups. DEP Ncr ${ }^{i \text { Cre/+}} R 26^{D T A /+}$ and $R 26^{D T A /+}$ littermate pups were generated as in Figure 3, A-J. After birth, pups were immunized and challenged with HDM and analyzed 72 hours after challenge. (A) Percentages of IL-5+ and IL-13+ NK cells in live lung NK cells (after ex vivo stimulation with PMA/ionomycin). $n=6$ per group. (B and I) Percentages of IL- $5^{+}$and IL-13+CD4+ T cells in live lung cells. $n=6$. (C and J) HDM-specific IgE in the serum. $n=9$. (D and K) Percentages of IL25R+ST2- ILC2s, IL25R+ST2+ ILC2s, and IL25R-ST2+ ILC2s in live lung cells. $n=5$ (D); $n=10$ (K). (E and L) Percentages of IL-5+ and IL-13 ${ }^{+}$ILC2s in live lung cells. $n=6$. (F and $\left.\mathbf{M}\right)$ Total lung resistance to methacholine. $n=6$. (G and $\left.\mathbf{N}\right)$ Leukocyte subset counts in BAL fluid. $n=5$ (G); $n=8$ (N). (H and $\mathbf{O})$ Peribronchial inflammation scores (left, $n=5$ ) and proportions of bronchial epithelial areas that are PAS $($ right, $n=5)$. Data are representative of 2 independent experiments and are shown as mean $\pm \mathrm{SEM}$. ${ }^{*} P<0.05 ;{ }^{* *} P<0.01 ;{ }^{* * *} P<0.001$, 1-way ANOVA with Tukey's post hoc test (A-E, G, and $\mathbf{H}$ ); 2-tailed unpaired $t$ test (I-L, $\mathbf{N}$, and $\mathbf{O}$ ); and 2-way repeated-measures ANOVA with Bonferroni's post hoc test (F and $\mathbf{M})$. 
A

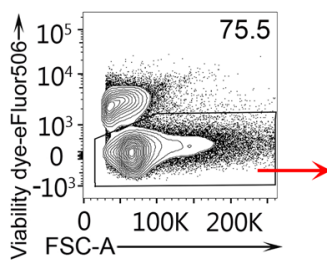

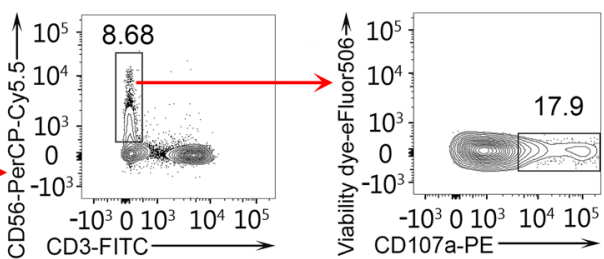

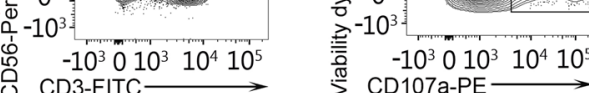

B

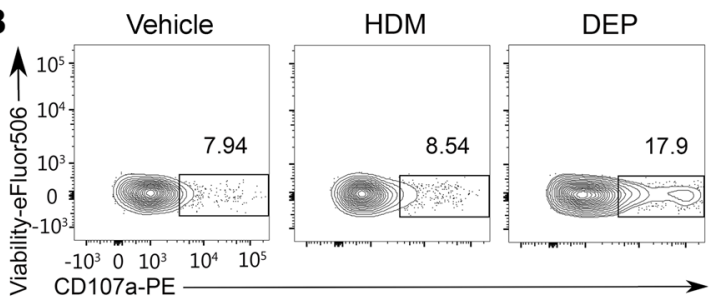

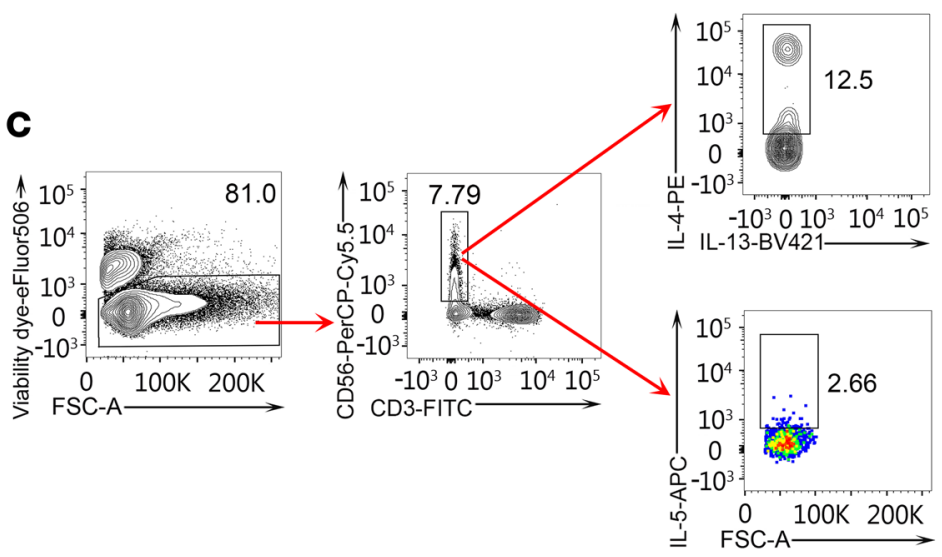

D
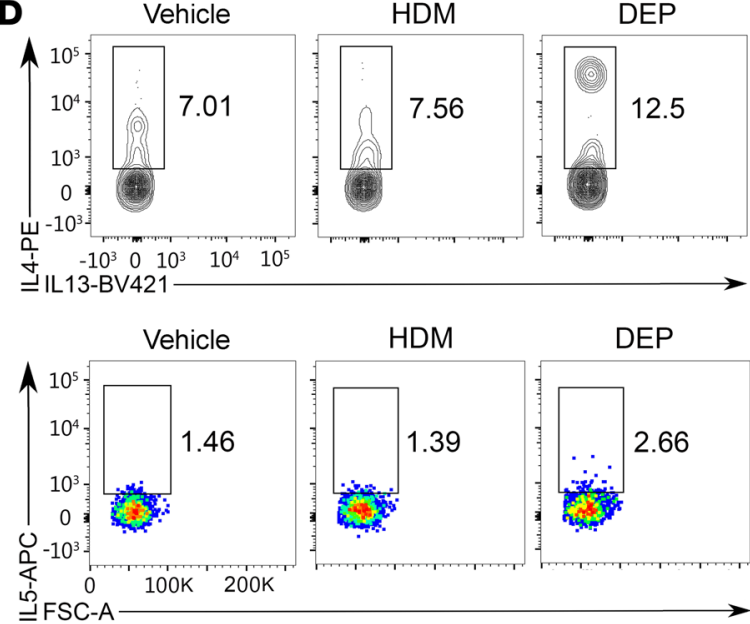

E

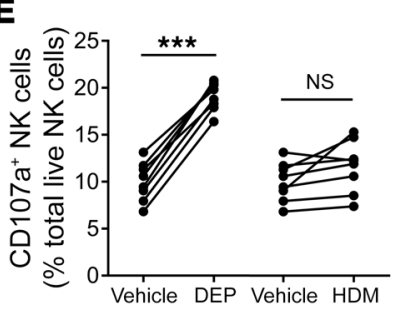

$\mathbf{F}$

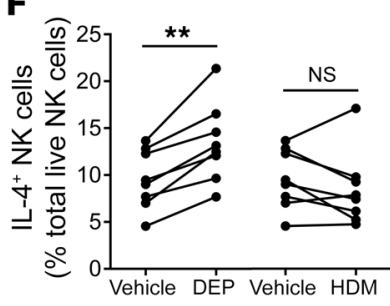

G

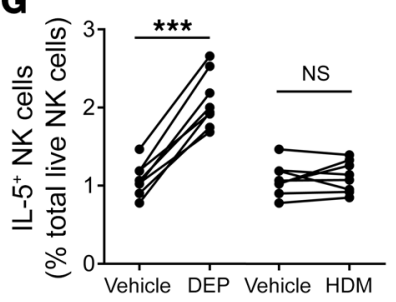

H

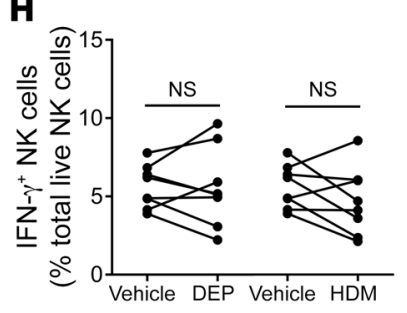

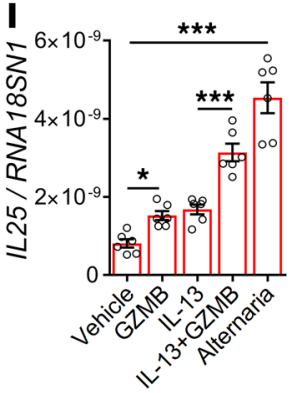

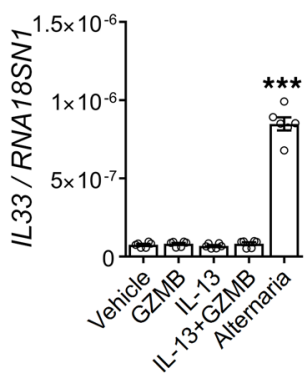

Figure 11. The NK cell pathway in human cell systems. (A-H) Human CBMCs were incubated with DEP, HDM, or vehicle (PBS) for 48 hours. To measure NK cell degranulation, PE-labeled anti-CD107a or isotype control IgG, monensin, and brefeldin A were then added for an additional 5 hours. To measure intracellular cytokines in NK cells, monensin and brefeldin A were added for an additional 4 hours. Cells were then stained with eFluor506 (viability) and antibodies for NK cell surface markers \pm antibodies for cytokines. (A and C) Gating strategy to define degranulated (A) and cytokine-producing (C) NK cells. Lymphocytes (from the FSC-A vs. SSC-A plot) were gated on singlets and then on live cells (eFluor506-). eFluor506- single lymphocytes were analyzed for CD56 and CD3. NK cells (CD56+CD3-) were analyzed for CD107a (marking degranulated cells; A) or intracellular cytokines (C). (B and D) Representative flow cytometry plots showing anti-CD107a labeling of NK cells (B) and anti-cytokine labeling of NK cells (D) under 3 stimulation conditions (DEP, HDM, and vehicle). (E-H) Percentages of degranulated (CD107a+) NK cells (E), IL-4+ NK cells (F), IL-5+ NK cells (G), and IFN- $\gamma^{+}$NK cells (H) in total live NK cells. $n=8$ subjects. (I) Levels of IL25 and IL33 mRNAs in human primary airway epithelial cells treated with vehicle (PBS), human granzyme B \pm human IL-13, human IL-13, or an extract of A. alternata. RNA18SN1, 18S ribosomal RNA. Data are pooled from 8 independent experiments (E-H) or are representative of 3 independent experiments (I). Data are shown as mean \pm SEM. ${ }^{*} P<0.05 ;{ }^{* *} P<0.01 ;{ }^{* * *} P<0.001$, 2-tailed paired $t$ test $(\mathbf{E}-\mathbf{H}) ; 1$-way ANOVA with Tukey's post hoc test (I).

in some circumstances, NK cells may participate in resolution of allergic inflammation (39). Here, we show that in the context of maternal DEP, NK cells become key drivers of the type 2 immune response, allergic inflammation, and AAD.
Granzyme B is best known for its capacity to kill targetcells. This occurs after its intracellular delivery via cleavage and activation of proapoptotic caspases $(25,26)$. More recent reports indicate that granzyme B may also have noncytotoxic functions. Granzyme B 
cleaves extracellular matrix proteins and thereby facilitates chemokine-induced movement of cytotoxic lymphocytes through basement membranes (40). Furthermore, granzyme B amplifies LPS-induced TNF- $\alpha$ release from human monocytes in vitro (41). Finally, granzyme B cleaves IL- $1 \alpha$, enhancing its biological activity (42). It remains to be established whether granzyme B-mediated TNF- $\alpha$ release and IL- $1 \alpha$ cleavage occur in vivo and whether these processes are important for inflammation and development of diseases. There are no reports, to our knowledge, that define specific functions of granzyme B in type 2 inflammation. We believe that our manuscript is an opening act in pursuit of this question. Intriguingly, the granzyme B-triggered mechanism that we uncovered resembles some of the mechanisms that are elicited by allergens. House dust mite and proallergic fungi (A. alternata and Aspergillus) contain proteases and induce asthma partly via PAR2-mediated activation of the airway epithelium (43).

The lung has the highest frequency of NK cells ( $10 \%$ of lymphocytes) among all nonlymphoid and lymphoid organs (44), suggesting that NK cells play special roles in the lung. NK cells defend against respiratory infections and destroy cigarette smokedamaged epithelial cells in chronic obstructive pulmonary disease (COPD) $(45,46)$. Whether NK cells have other functions in the lung is not clear. Here, we propose that, fueled by maternal exposure to DEP, NK cells become critically engaged in responses to inhaled allergens. By controlling production of type 2 mediators by the airway epithelium, NK cells contribute to induction of pulmonary type 2 responses and AAD.

The importance of NK cells in mouse models of AAD was explored in previous studies, but the results were conflicting (47$49)$. One potential reason for lack of clarity was the use of nonspecific tools for NK cell depletion. To address this, we used the $N c r 1^{i C r e} R 26^{\text {DTA }}$ strain of mice, representing the most specific model of NK cell deficiency to date. We then complemented these studies through the use of NK cell-depleting antibodies anti-asialoGM1 (this manuscript) and anti-NK1.1 (14) and by performing the NK cell reconstitution experiment. All experiments led to the same conclusion - NK cells drove AAD in our DEP-dependent model. We also observed that NK cells were redundant in models that rely exclusively on allergens to elicit AAD. This is consistent with a recent publication that used $N c r 1^{i \text { Cre }} R 26^{D T A}$ mice to report no role of NK cells in adult allergen-driven AAD (22).

NK cell priming in our model may have several causes that likely act in concert. The first likely causes are cellular stress and cell damage that are triggered by transplacentally transferred DEP and DEP-derived compounds or by endogenous molecules, including cytokines that are overproduced by DEP-challenged mothers and fetuses. Prior data provide a rationale to hypothesize that DEP activity extends beyond the exposure period (preconception) and into the pregnancy. DEP and DEP-derived polylic aromatic hydrocarbons (PAH) have been shown to persist in the body long after cessation of the exposure (50). PAH are known to cross the placental barrier (51). DEPs are likely to have this capacity; a large proportion of DEPs are nanoparticles (52), and nanoparticles, in general, are easily transferable via the placenta (53). Transplacentally transferred DEP, PAH, and DEP/PAH-induced endogenous molecules may stress or damage offspring cells. Stressed and damaged cells are known to activate NK cells through upregulation of NKG2D and DNAM-1 ligands, downregulation of MHCI, and production of NK cell-stimulatory cytokines. Some of these molecules are linked to type 2 immunity. A previous study shows that physical damage of the epidermis (tape stripping) results in upregulation of NKG2D ligands on keratinocytes (54). Under these circumstances, epidermal NKG2D ligands promote type 2 cytokine production by NKG2D-expressing intraepithelial lymphocytes; if skin damage is followed by allergen application to the damaged area, NKG2D ${ }^{+}$type 2 cytokine-expressing lymphocytes initiate the type 2 immune response to this allergen, leading to induction of allergen-specific IgE. NK cells may also be primed by DEP-derived compounds. NK cells express aryl hydrocarbon receptor (AHR), an environment-sensing transcriptional factor that is targeted by DEP-derived PAH. AHR promotes NK cell survival (55) and cytolytic activity (56). In other cell types, such as mast cells and $\mathrm{T}$ cells, PAH and AHR induce type 2 cytokines (57), suggesting that type 2 programming of NK cells in our model may be a result of AHR activation. We have evidence that the AHR pathway is activated in DEP-exposed mothers and their offspring $(13,14)$.

Finally, we would like to comment on some of our results on ILC2s/Th2s. When comparing our data on IL-25 with data on IL-25 targets (IL25R $\mathrm{ILC}^{+} \mathrm{s} / \mathrm{Th} 2 \mathrm{~s}$ ), we noticed an interesting discrepancy: DEP-PBS pups had moderately increased frequencies of these cells despite having normal levels of IL-25 (Figure 1, E, H, and J, and Figure $4, \mathrm{~A}$ and B). We speculate that moderate expansion of DEP-PBS IL25R $\mathrm{R}^{+}$ILC2s and Th2 cells may be due to increased responsiveness of these cells to IL-25. Alternatively, this may be due to another DEP-regulated mediator that specifically targets IL $25 \mathrm{R}^{+}$ILC2s and Th2 cells. We will address these hypotheses in another study.

Taken together, our studies provide proof of concept that the NK cell/airway epithelium axis plays an important role in DEP-triggered transmission of asthma predisposition from the mother to her offspring. We hope that the identified principles will facilitate development of new diagnostic tools for early detection and precise classification of asthma.

\section{Methods}

Additional information can be found in the Supplemental Methods.

Statistics. To compare the means of 2 matched groups and the means of 2 unmatched groups, 2 -tailed paired and unpaired $t$ tests were used, respectively. To compare the means of 3 or more unmatched groups, 1-way ANOVA was performed, followed by Tukey's or Dunnett's post hoc tests. To analyze data with 2 or more groups and repeated measurements on individual mice (FlexiVent studies), 2-way repeated-measures ANOVA was used, followed by Bonferroni's post hoc test. Data are presented as mean \pm SEM. Statistical significance was defined as $P<0.05$. For box plots, lines within the boxes represent medians, boxes represent 25th to 75 th percentiles, and whiskers represent minimum and maximum values. All statistical analysis was performed using Prism software (GraphPad 7).

Study approval. All procedures on animals were reviewed and approved by the IACUC at NJH (IACUC AS2798-12-20). All studies on human samples were reviewed and approved by the IRB at NJH (IRB HS-3509 for the cord blood study and IRB HS-2604 and HS-3114 for collection of bronchial brushings). Cord blood samples were received from the University of Colorado Cord Blood Bank (Aurora, Colorado, USA). Bronchial brushings were collected during bronchoscopy by the NJH 
Biobank Honest Broker System. Brushings were then transferred to the Human Primary Cell Culture Core at NJH, from which the samples were distributed to the study investigators. Before subject enrollment and sample collection, written, informed consent was obtained from all subjects. Investigators in this study were not involved in subject enrollment, consent, and sample collection and were blinded to donor identities.

\section{Author contributions}

$\mathrm{QQ}, \mathrm{BPC}, \mathrm{ZS}$, and JL performed experiments and analyzed data. MMG conceived and directed the study, designed experiments, and analyzed data. MMG and QQ prepared figures and wrote the manuscript. RA provided helpful discussion and reviewed the manuscript. EV generated a key mouse strain $\left(\mathrm{Ncr}^{\mathrm{iCre} /+}\right)$ and reviewed the manuscript.

\section{Acknowledgments}

This work was supported by NIH grant R01HL122995 and ALA Biomedical Research Grant RG-310463 (both to MMG). EV gener- ated $\mathrm{Ncr}^{\mathrm{iCre} /+}$ mice and is supported by funding from the European Research Council (ERC) under the European Union's Horizon 2020 Research and Innovation Program (TILC, grant agreement 694502); the Agence Nationale de la Recherche, Equipe Labellisée "La Ligue," Ligue Nationale contre le Cancer; MSDAvenir; Innate Pharma; and institutional grants to the CIML (INSERM, CNRS, and Aix-Marseille University) and to Marseille Immunopôle. We thank Joseph Sun (Memorial Sloan Kettering Cancer Center, New York, New York, USA) for sending $\mathrm{Ncr}^{\mathrm{iCre} /+}$ mice, Timothy J. Ley (Washington University School of Medicine, St. Louis, Missouri, USA) for generation and permission to use his $\mathrm{Gzmb}^{-/-}$mice, and Xuefang Cao (Roswell Park Comprehensive Cancer Center, Buffalo, New York, USA) for sending $\mathrm{Gzmb}^{-/-}$mice.

Address correspondence to: Magdalena M. Gorska, Division of Allergy and Clinical Immunology, Department of Medicine, National Jewish Health, 1400 Jackson Street, Denver, Colorado 80206, USA; Phone: 303.398.1656; Email: gorskam@njhealth.org.
1. Bisgaard H, Bønnelykke K. Long-term studies of the natural history of asthma in childhood. J Allergy Clin Immunol. 2010;126(2):187-197.

2. Vercelli D. Does epigenetics play a role in human asthma? Allergol Int. 2016;65(2):123-126.

3. Igartua C, et al. Ethnic-specific associations of rare and low-frequency DNA sequence variants with asthma. Nat Commun. 2015;6:5965.

4. von Mutius E, Martinez FD, Fritzsch C, Nicolai T, Roell G, Thiemann HH. Prevalence of asthma and atopy in two areas of West and East Germany. Am J Respir Crit Care Med. 1994;149(2 pt 1):358-364.

5. Riedler J, Eder W, Oberfeld G, Schreuer M. Austrian children living on a farm have less hay fever, asthma and allergic sensitization. Clin Exp Allergy. 2000;30(2):194-200.

6. Brandt EB, Myers JM, Ryan PH, Hershey GK. Air pollution and allergic diseases. Curr Opin Pediatr. 2015;27(6):724-735.

7. Illi S, et al. Protection from childhood asthma and allergy in Alpine farm environments-the GABRIEL Advanced Studies. JAllergy Clin Immunol. 2012;129(6):1470-1477.e6.

8. McConnell R, et al. Childhood incident asthma and traffic-related air pollution at home and school. Environ Health Perspect. 2010;118(7):1021-1026.

9. Ryan PH, et al. Is it traffic type, volume, or distance? Wheezing in infants living near truck and bus traffic. J Allergy Clin Immunol. 2005;116(2):279-284.

10. Muranaka M, et al. Adjuvant activity of dieselexhaust particulates for the production of IgE antibody in mice. J Allergy Clin Immunol. 1986;77(4):616-623.

11. Hsu HH, et al. Prenatal particulate air pollution and asthma onset in urban children. Identifying sensitive windows and sex differences. Am J Respir Crit Care Med. 2015;192(9):1052-1059.

12. Jedrychowski WA, et al. Intrauterine exposure to polycyclic aromatic hydrocarbons, fine particulate matter and early wheeze. Prospective birth cohort study in 4-year olds. Pediatr Allergy Immunol. 2010;21(4 Pt 2):e723-e732.
13. Lenberg J, Qian Q, Sun Z, Alam R, Gorska MM. Pre-pregnancy exposure to diesel exhaust predisposes offspring to asthma through IL-1 $\beta$ and IL-17A. J Allergy Clin Immunol. 2018;141(3):1118-1122.e3.

14. Manners S, Alam R, Schwartz DA, Gorska MM. A mouse model links asthma susceptibility to prenatal exposure to diesel exhaust. J Allergy Clin Immunol. 2014;134(1):63-72.

15. Chiossone L, Chaix J, Fuseri N, Roth C, Vivier E, Walzer T. Maturation of mouse NK cells is a 4-stage developmental program. Blood. 2009;113(22):5488-5496.

16. Sun JC, Beilke JN, Lanier LL. Adaptive immune features of natural killer cells. Nature. 2009;457(7229):557-561.

17. Narni-Mancinelli E, et al. Fate mapping analysis of lymphoid cells expressing the NKp46 cell surface receptor. Proc Natl Acad Sci U S A. 2011;108(45):18324-18329.

18. Bezman NA, et al. Molecular definition of the identity and activation of natural killer cells. Nat Immunol. 2012;13(10):1000-1009.

19. Rankin LC, et al. Complementarity and redundancy of IL-22-producing innate lymphoid cells. Nat Immunol. 2016;17(2):179-186.

20. Matsumoto A, et al. IL-22-producing ROR $\gamma \mathrm{t}$ dependent innate lymphoid cells play a novel protective role in murine acute hepatitis. PLOS One. 2013;8(4):e62853.

21. Vonarbourg C, et al. Regulated expression of nuclear receptor ROR $\gamma \mathrm{t}$ confers distinct functional fates to NK cell receptor-expressing ROR $\gamma \mathrm{t}(+)$ innate lymphocytes. Immunity. 2010;33(5):736-751.

22. Haspeslagh E, et al. Role of $\mathrm{NKp} 46^{+}$natural killer cells in house dust mite-driven asthma. EMBO MolMed. 2018;10(4):e8657.

23. Zhao A, et al. Critical role of IL-25 in nematode infection-induced alterations inintestinal function. J Immunol. 2010;185(11):6921-6929.

24. Hanna J, Bechtel P, Zhai Y, Youssef F, McLachlan $\mathrm{K}$, Mandelboim $\mathrm{O}$. Novel insights on human NK cells' immunological modalities revealed by gene expression profiling. JImmunol.
2004;173(11):6547-6563

25. Voskoboinik I, Whisstock JC, Trapani JA. Perforin and granzymes: function, dysfunction and human pathology. Nat Rev Immunol. 2015;15(6):388-400.

26. Froelich CJ, et al. New paradigm for lymphocyte granule-mediated cytotoxicity. Target cells bind and internalize granzyme $\mathrm{B}$, but an endosomolytic agent is necessary for cytosolic delivery and subsequent apoptosis. J Biol Chem. 1996;271(46):29073-29079.

27. Molofsky AB, Savage AK, Locksley RM. Interleukin-33 in tissue homeostasis, injury, and inflammation. Immunity. 2015;42(6):1005-1019.

28. Gerbe F, et al. Intestinal epithelial tuft cells initiate type 2 mucosal immunity to helminth parasites. Nature. 2016;529(7585):226-230.

29. Howitt MR, et al. Tuft cells, taste-chemosensory cells, orchestrate parasite type 2 immunity in the gut. Science. 2016;351(6279):1329-1333.

30. von Moltke J, Ji M, Liang HE, Locksley RM. Tuft-cell-derived IL-25 regulates an intestinal ILC2-epithelial response circuit. Nature. 2016;529(7585):221-225.

31. Kakkar R, Hei H, Dobner S, Lee RT. Interleukin 33 as a mechanically responsive cytokine secreted by living cells. J Biol Chem. 2012;287(9):6941-6948.

32. Kouzaki H, Iijima K, Kobayashi T, O'Grady SM, Kita H. The danger signal, extracellular ATP, is a sensor for an airborne allergen and triggers IL-33 release and innate Th2-type responses. JImmunol. 2011;186(7):4375-4387.

33. Altman MC, et al. Allergen-induced activation of natural killer cells represents an earlylife immune response in the development of allergic asthma. J Allergy Clin Immunol. 2018;142(6):1856-1866.

34. Yang IV, et al. DNA methylation and childhood asthma in the inner city. J Allergy Clin Immunol. 2015;136(1):69-80.

35. Hong JY, et al. Neonatal rhinovirus induces mucous metaplasia and airways hyperresponsiveness through IL-25 and type 2 innate lymphoid cells. J Allergy Clin Immunol. 
2014;134(2):429-439.

36. Sadeghnejad A, Karmaus W, Davis S, Kurukulaaratchy RJ, Matthews S, Arshad SH. Raised cord serum immunoglobulin $\mathrm{E}$ increases the risk of allergic sensitisation at ages 4 and 10 and asthma at age 10. Thorax. 2004;59(11):936-942.

37. Piccioli D, Sbrana S, Melandri E, Valiante NM. Contact-dependent stimulation and inhibition of dendritic cells by natural killer cells. J Exp Med. 2002;195(3):335-341.

38. Wei H, Zhang J, Xiao W, Feng J, Sun R, Tian Z. Involvement of human natural killer cells in asthma pathogenesis: natural killer 2 cells in type 2 cytokine predominance. J Allergy Clin Immunol. 2005;115(4):841-847.

39. Barnig C, et al. Lipoxin A4 regulates natural killer cell and type 2 innate lymphoid cell activation in asthma. Sci Transl Med. 2013;5(174):174ra26.

40. Prakash MD, et al. Granzyme B promotes cytotoxic lymphocyte transmigration via basement membrane remodeling. Immunity. 2014;41(6):960-972.

41. Wensink AC, et al. Granzyme K synergistically potentiates LPS-induced cytokine responses in human monocytes. Proc Natl Acad Sci US A. 2014;111(16):5974-5979.

42. Afonina IS, et al. Granzyme B-dependent proteolysis acts as a switch to enhance the proinflammatory activity of IL-1 $\alpha$. Mol Cell. 2011;44(2):265-278.

43. Asokananthan $\mathrm{N}$, et al. House dust mite aller- gens induce proinflammatory cytokines from respiratory epithelial cells: the cysteine protease allergen, Der $\mathrm{p} 1$, activates protease-activated receptor (PAR)-2 and inactivates PAR-1. J Immunol. 2002;169(8):4572-4578.

44. Grégoire C, et al. The trafficking of natural killer cells. Immunol Rev. 2007;220:169-182.

45. Zhou G, Juang SW, Kane KP. NK cells exacerbate the pathology of influenza virus infection in mice. Eur J Immunol. 2013;43(4):929-938.

46. Motz GT, et al. Chronic cigarette smoke exposure primes NK cell activation in a mouse model of chronic obstructive pulmonary disease. J Immunol. 2010;184(8):4460-4469.

47. Haworth O, Cernadas M, Levy BD. NK cells are effectors for resolvin $\mathrm{E} 1$ in the timely resolution of allergic airway inflammation. J Immunol. 2011;186(11):6129-6135.

48. Korsgren M, et al. Natural killer cells determine development of allergen-induced eosinophilic airway inflammation in mice. J Exp Med. 1999;189(3):553-562.

49. Mathias CB, et al. Pro-inflammatory role of natural killer cells in the development of allergic airway disease. Clin Exp Allergy. 2014;44(4):589-601.

50. Gerde P, Muggenburg BA, Lundborg M, Dahl AR. The rapid alveolar absorption of diesel sootadsorbed benzo[a]pyrene: bioavailability, metabolism and dosimetry of an inhaled particle-borne carcinogen. Carcinogenesis. 2001;22(5):741-749.
51. Srivastava VK, Chauhan SS, Srivastava PK, Kumar V, Misra UK. Fetal translocation and metabolism of PAH obtained from coal fly ash given intratracheally to pregnant rats. J Toxicol Environ Health. 1986;18(3):459-469.

52. Li N, et al. A work group report on ultrafine particles (American Academy of Allergy, Asthma \& Immunology): Why ambient ultrafine and engineered nanoparticles should receive special attention for possible adverse health outcomes in human subjects. JAllergy Clin Immunol. 2016;138(2):386-396.

53. Wick P, et al. Barrier capacity of human placenta for nanosized materials. Environ Health Perspect. 2010;118(3):432-436.

54. Strid J, Sobolev O, Zafirova B, Polic B, Hayday A. The intraepithelial $T$ cell response to NKG2Dligands links lymphoid stress surveillance to atopy. Science. 2011;334(6060):1293-1297.

55. Zhang LH, Shin JH, Haggadone MD, Sunwoo JB. The aryl hydrocarbon receptor is required for the maintenance of liver-resident natural killer cells. JExp Med. 2016;213(11):2249-2257.

56. Shin JH, et al. Modulation of natural killer cell antitumor activity by the aryl hydrocarbon receptor. Proc Natl Acad Sci U S A. 2013;110(30):12391-12396.

57. Bömmel H, Li-Weber M, Serfling E, Duschl A. The environmental pollutant pyrene induces the production of IL-4. J Allergy Clin Immunol. 2000;105(4):796-802. 